\title{
Design of TTF-based phosphazenes combining a good electron-donor capacity and possible inclusion adduct formation
}

\author{
Godefroid Gahungu, Bin Zhang, Jingping Zhang.
}

\section{Supporting materials}

\section{Index}

1. Cartesian coordinates of the relevant structures

Pages $2-53$

2. Figures (S1, S2, S3) not given in the text

Pages $54-55$

3. Tables (S1 to S6) listing the numerical results not given in the text

Pages $56-63$ 


\section{Cartesian coordinates of the relevant structures}

$\operatorname{TTF}(d)$

$\begin{array}{rrrc}\mathrm{C} & 0.674847 & 0.000038 & 0.000218 \\ \mathrm{C} & -0.674847 & -0.000038 & 0.000218 \\ \mathrm{~S} & -1.651349 & -1.499036 & 0.000238 \\ \mathrm{~S} & 1.651349 & 1.499036 & 0.000238 \\ \mathrm{~S} & -1.651422 & 1.498905 & 0.000209 \\ \mathrm{~S} & 1.651422 & -1.498905 & 0.000209 \\ \mathrm{C} & -3.205503 & -0.668594 & 0.000209 \\ \mathrm{C} & 3.205503 & 0.668594 & 0.000209 \\ \mathrm{C} & -3.205538 & 0.668420 & 0.000190 \\ \mathrm{C} & 3.205538 & -0.668420 & 0.000190 \\ \mathrm{H} & -4.095329 & -1.287276 & 0.000205 \\ \mathrm{H} & 4.095329 & 1.287276 & 0.000205 \\ \mathrm{H} & -4.095383 & 1.287062 & 0.000120 \\ \mathrm{H} & 4.095383 & -1.287062 & 0.000120\end{array}$

$\operatorname{TMTSF}(d)$

$\begin{array}{cccc}\mathrm{C} & 0.001964 & -0.238421 & -0.671255 \\ \mathrm{C} & 0.002106 & -0.238420 & 0.671255 \\ \mathrm{Se} & 1.586118 & -0.239133 & -1.728798 \\ \mathrm{Se} & -1.581805 & -0.266171 & 1.728798 \\ \mathrm{C} & 0.667816 & 0.356339 & -3.311132 \\ \mathrm{C} & -0.673801 & 0.344888 & 3.311132 \\ \mathrm{C} & -0.673242 & 0.346313 & -3.310907 \\ \mathrm{C} & 0.667233 & 0.357754 & 3.310907 \\ \mathrm{Se} & -1.582280 & -0.262769 & -1.728516 \\ \mathrm{Se} & 1.586535 & -0.235724 & 1.728516 \\ \mathrm{C} & 1.581767 & 0.771002 & -4.430589 \\ \mathrm{C} & -1.594697 & 0.743892 & 4.430589 \\ \mathrm{H} & 1.030979 & 0.999813 & -5.346443 \\ \mathrm{H} & -1.047895 & 0.982070 & 5.346443 \\ \mathrm{H} & 2.162747 & 1.659517 & -4.153989 \\ \mathrm{H} & -2.190758 & 1.622360 & 4.153989 \\ \mathrm{H} & 2.298901 & -0.026608 & -4.664556 \\ \mathrm{H} & -2.298112 & -0.065843 & 4.664556 \\ \mathrm{C} & -1.592966 & 0.748107 & -4.430336 \\ \mathrm{C} & 1.579965 & 0.775188 & 4.430336 \\ \mathrm{H} & -2.294951 & -0.061596 & -4.668687 \\ \mathrm{H} & 2.295668 & -0.022416 & 4.668687 \\ \mathrm{H} & -2.190605 & 1.624714 & -4.151322 \\ \mathrm{H} & 2.162554 & 1.661867 & 4.151322 \\ \mathrm{H} & -1.044622 & 0.991016 & -5.343995 \\ \mathrm{H} & 1.027554 & 1.008702 & 5.343995\end{array}$






BETS-TTF

$\begin{array}{rrrl}\text { C } & 0.671406 & 0.001796 & -0.534557 \\ \text { C } & -0.671406 & -0.001796 & -0.534557 \\ \text { Se } & 1.731889 & -1.585494 & -0.576694 \\ \text { Se } & -1.731889 & 1.585494 & -0.576694 \\ \text { Se } & 1.739632 & 1.582602 & -0.557080 \\ \text { Se } & -1.739632 & -1.582602 & -0.557080 \\ \text { C } & 3.200999 & -0.684067 & 0.281103 \\ \text { C } & -3.200999 & 0.684067 & 0.281103 \\ \text { C } & 3.201139 & 0.661852 & 0.284355 \\ \text { C } & -3.201139 & -0.661852 & 0.284355 \\ \text { S } & 4.411708 & -1.775286 & 0.953101 \\ \text { S } & -4.411708 & 1.775286 & 0.953101 \\ \text { C } & 5.973086 & -0.764873 & 0.977459 \\ \text { C } & -5.973086 & 0.764873 & 0.977459 \\ \text { C } & 5.896185 & 0.565652 & 0.242127 \\ \text { C } & -5.896185 & -0.565652 & 0.242127 \\ \text { S } & 4.542231 & 1.647656 & 0.865168\end{array}$




$\begin{array}{rrrr}\mathrm{S} & -4.542231 & -1.647656 & 0.865168 \\ \mathrm{H} & 6.226997 & -0.602197 & 2.027190 \\ \mathrm{H} & -6.226997 & 0.602197 & 2.027190 \\ \mathrm{H} & 6.744460 & -1.396170 & 0.522278 \\ \mathrm{H} & -6.744460 & 1.396170 & 0.522278 \\ \mathrm{H} & 6.826169 & 1.120938 & 0.401740 \\ \mathrm{H} & -6.826169 & -1.120938 & 0.401740 \\ \mathrm{H} & 5.752796 & 0.426402 & -0.832551 \\ \mathrm{H} & -5.752796 & -0.426402 & -0.832551\end{array}$

BEDO-TTF

$\begin{array}{lrrr}\mathrm{O} & 0.000000 & 0.000000 & 0.000000 \\ \mathrm{C} & 0.000000 & 0.000000 & 1.368275 \\ \mathrm{C} & 1.105610 & 0.000000 & 2.127685 \\ \mathrm{O} & 2.383520 & -0.039077 & 1.641721 \\ \mathrm{C} & 2.389898 & -0.382624 & 0.248811 \\ \mathrm{C} & 1.292119 & 0.374230 & -0.498757 \\ \mathrm{~S} & -1.573662 & 0.101628 & 2.179579 \\ \mathrm{C} & -0.858479 & -0.142021 & 3.799562 \\ \mathrm{~S} & 0.911409 & 0.098250 & 3.888241 \\ \mathrm{C} & -1.602336 & -0.452455 & 4.883714 \\ \mathrm{~S} & -3.379191 & -0.638979 & 4.805645 \\ \mathrm{C} & -3.457754 & -1.432908 & 6.390089 \\ \mathrm{C} & -2.350514 & -1.445441 & 7.147017 \\ \mathrm{~S} & -0.891216 & -0.664760 & 6.509882 \\ \mathrm{O} & -2.284182 & -1.957137 & 8.414277 \\ \mathrm{C} & -3.602865 & -2.126229 & 8.953533 \\ \mathrm{C} & -4.516137 & -2.793078 & 7.924917 \\ \mathrm{O} & -4.661838 & -1.963350 & 6.763630 \\ \mathrm{H} & -5.522585 & -2.938763 & 8.324251 \\ \mathrm{H} & 3.380736 & -0.116472 & -0.126730 \\ \mathrm{H} & -4.095579 & -3.761859 & 7.626483 \\ \mathrm{H} & 2.235046 & -1.463915 & 0.142525 \\ \mathrm{H} & -4.008520 & -1.146672 & 9.237303 \\ \mathrm{H} & 1.430617 & 1.456016 & -0.375582 \\ \mathrm{H} & -3.491869 & -2.749256 & 9.843918 \\ \mathrm{H} & 1.294475 & 0.122756 & -1.561784\end{array}$

Molecule 1a

$\begin{array}{rrrr}\text { C } & 0.00099 & 1.34883 & 0.33078 \\ \mathrm{O} & -1.16531 & 0.67368 & 0.60285 \\ \mathrm{C} & -1.16861 & -0.67356 & 0.33078 \\ \mathrm{O} & -0.00077 & -1.34603 & 0.60285 \\ \mathrm{C} & 1.16763 & -0.67527 & 0.33078 \\ \mathrm{O} & 1.16608 & 0.67234 & 0.60285 \\ \mathrm{C} & 0.00000 & 2.62544 & -0.07118\end{array}$




\begin{tabular}{|c|c|c|c|}
\hline $\mathrm{C}$ & -2.27369 & -1.31272 & -0.07118 \\
\hline $\mathrm{C}$ & 2.27369 & -1.31272 & -0.07118 \\
\hline $\mathrm{S}$ & 1.48658 & 3.55679 & -0.36287 \\
\hline $\mathrm{S}$ & -3.82356 & -0.49098 & -0.36287 \\
\hline $\mathrm{S}$ & 2.33699 & -3.06581 & -0.36287 \\
\hline $\mathrm{C}$ & 0.67137 & 5.14731 & -0.30427 \\
\hline $\mathrm{C}$ & -4.79339 & -1.99223 & -0.30427 \\
\hline $\mathrm{C}$ & 4.12202 & -3.15508 & -0.30427 \\
\hline $\mathrm{C}$ & -0.68377 & 5.14390 & -0.30697 \\
\hline $\mathrm{C}$ & -4.11286 & -3.16411 & -0.30697 \\
\hline $\mathrm{C}$ & 4.79663 & -1.97978 & -0.30697 \\
\hline $\mathrm{S}$ & -1.49048 & 3.54934 & -0.36784 \\
\hline $\mathrm{S}$ & -2.32858 & -3.06546 & -0.36784 \\
\hline $\mathrm{S}$ & 3.81906 & -0.48388 & -0.36784 \\
\hline $\mathrm{S}$ & 1.69810 & 6.58255 & -0.40455 \\
\hline $\mathrm{S}$ & -6.54970 & -1.82068 & -0.40455 \\
\hline $\mathrm{S}$ & 4.85160 & -4.76187 & -0.40455 \\
\hline $\mathrm{S}$ & -1.71665 & 6.57406 & -0.41567 \\
\hline $\mathrm{S}$ & -4.83498 & -4.77369 & -0.41567 \\
\hline $\mathrm{S}$ & 6.55162 & -1.80037 & -0.41567 \\
\hline $\mathrm{C}$ & 2.68283 & 6.44316 & 1.14129 \\
\hline $\mathrm{C}$ & -6.92136 & -0.89818 & 1.14129 \\
\hline $\mathrm{C}$ & 4.23853 & -5.54498 & 1.14129 \\
\hline $\mathrm{C}$ & -2.72069 & 6.42648 & 1.11693 \\
\hline $\mathrm{C}$ & -4.20515 & -5.56942 & 1.11693 \\
\hline $\mathrm{C}$ & 6.92584 & -0.85706 & 1.11693 \\
\hline $\mathrm{H}$ & 3.37274 & 7.29131 & 1.12937 \\
\hline $\mathrm{H}$ & -8.00083 & -0.72478 & 1.12937 \\
\hline $\mathrm{H}$ & 4.62809 & -6.56653 & 1.12937 \\
\hline $\mathrm{H}$ & 2.03670 & 6.51425 & 2.01896 \\
\hline $\mathrm{H}$ & -6.65985 & -1.49329 & 2.01896 \\
\hline $\mathrm{H}$ & 4.62316 & -5.02096 & 2.01896 \\
\hline $\mathrm{H}$ & 3.25553 & 5.51405 & 1.16884 \\
\hline $\mathrm{H}$ & -6.40307 & 0.06234 & 1.16884 \\
\hline $\mathrm{H}$ & 3.14754 & -5.57639 & 1.16884 \\
\hline $\mathrm{H}$ & -3.42328 & 7.26386 & 1.09125 \\
\hline $\mathrm{H}$ & -4.57905 & -6.59657 & 1.09125 \\
\hline $\mathrm{H}$ & 8.00232 & -0.66728 & 1.09125 \\
\hline $\mathrm{H}$ & -3.27964 & 5.48890 & 1.14051 \\
\hline $\mathrm{H}$ & -3.11371 & -5.58470 & 1.14051 \\
\hline $\mathrm{H}$ & 6.39335 & 0.09580 & 1.14051 \\
\hline $\mathrm{H}$ & -2.08866 & 6.51096 & 2.00367 \\
\hline $\mathrm{H}$ & -4.59433 & -5.06431 & 2.00367 \\
\hline $\mathrm{H}$ & 6.68299 & -1.44665 & 2.00367 \\
\hline
\end{tabular}

Molecule -1b 


$\begin{array}{lrrr}\mathrm{C} & 0.52618 & 1.23756 & 0.57236 \\ \mathrm{O} & -0.81028 & 1.07646 & 0.86372 \\ \mathrm{C} & -1.33485 & -0.16310 & 0.57236 \\ \mathrm{O} & -0.52710 & -1.23995 & 0.86372 \\ \mathrm{C} & 0.80867 & -1.07446 & 0.57236 \\ \mathrm{O} & 1.33738 & 0.16350 & 0.86372 \\ \mathrm{C} & 1.02573 & 2.41276 & 0.17319 \\ \mathrm{C} & -2.60238 & -0.31807 & 0.17319 \\ \mathrm{C} & 1.57664 & -2.09469 & 0.17319 \\ \mathrm{~S} & 0.00000 & 3.85080 & -0.08567 \\ \mathrm{~S} & -3.33489 & -1.92540 & -0.08567 \\ \mathrm{~S} & 3.33489 & -1.92540 & -0.08567 \\ \mathrm{C} & 1.34810 & 4.88028 & -0.57489 \\ \mathrm{C} & -4.90050 & -1.27265 & -0.57489 \\ \mathrm{C} & 3.55240 & -3.60763 & -0.57489 \\ \mathrm{C} & 2.57802 & 4.35739 & -0.57572 \\ \mathrm{C} & -5.06262 & 0.05394 & -0.57572 \\ \mathrm{C} & 2.48459 & -4.41133 & -0.57572 \\ \mathrm{~S} & 2.77262 & 2.67192 & -0.08770 \\ \mathrm{~S} & -3.70026 & 1.06520 & -0.08770 \\ \mathrm{~S} & 0.92764 & -3.73712 & -0.08770 \\ \mathrm{H} & 1.10970 & 5.90064 & -0.85193 \\ \mathrm{H} & -5.66495 & -1.98929 & -0.85193 \\ \mathrm{H} & 4.55525 & -3.91135 & -0.85193 \\ \mathrm{H} & 3.47781 & 4.89392 & -0.85359 \\ \mathrm{H} & -5.97716 & 0.56492 & -0.85359 \\ \mathrm{H} & 2.49935 & -5.45883 & -0.85359\end{array}$

Molecule 1c

$\begin{array}{lrrr}\mathrm{C} & 0.57512 & 1.18944 & 1.07831 \\ \mathrm{O} & -0.75452 & 1.11022 & 1.45099 \\ \mathrm{C} & -1.31764 & -0.09665 & 1.07831 \\ \mathrm{O} & -0.58422 & -1.20855 & 1.45099 \\ \mathrm{C} & 0.74252 & -1.09279 & 1.07831 \\ \mathrm{O} & 1.33874 & 0.09833 & 1.45099 \\ \mathrm{C} & 1.07738 & 2.22872 & 0.41639 \\ \mathrm{C} & -2.46882 & -0.18132 & 0.41639 \\ \mathrm{C} & 1.39143 & -2.04740 & 0.41639 \\ \mathrm{Se} & 2.91390 & 2.30954 & -0.09916 \\ \mathrm{Se} & -3.45707 & 1.36874 & -0.09916 \\ \mathrm{Se} & 0.54317 & -3.67828 & -0.09916 \\ \mathrm{C} & 2.67648 & 4.00371 & -0.92831 \\ \mathrm{C} & -4.80555 & 0.31604 & -0.92831 \\ \mathrm{C} & 2.12908 & -4.31975 & -0.92831 \\ \mathrm{C} & 1.47451 & 4.58489 & -0.92766 \\ \mathrm{C} & -4.70789 & -1.01548 & -0.92766\end{array}$




$\begin{array}{lrrr}\mathrm{C} & 3.23337 & -3.56941 & -0.92766 \\ \mathrm{Se} & 0.00000 & 3.71883 & -0.09713 \\ \mathrm{Se} & -3.22060 & -1.85941 & -0.09713 \\ \mathrm{Se} & 3.22060 & -1.85941 & -0.09713 \\ \mathrm{H} & 3.55667 & 4.44479 & -1.38395 \\ \mathrm{H} & -5.62763 & 0.85778 & -1.38395 \\ \mathrm{H} & 2.07096 & -5.30256 & -1.38395 \\ \mathrm{H} & 1.27320 & 5.54893 & -1.38264 \\ \mathrm{H} & -5.44212 & -1.67185 & -1.38264 \\ \mathrm{H} & 4.16892 & -3.87709 & -1.38264\end{array}$

Molecule 1d

$\begin{array}{lrrr}\mathrm{C} & 0.18581 & 1.33543 & 1.18007 \\ \mathrm{O} & 1.24768 & 0.50600 & 1.45590 \\ \mathrm{C} & 1.06361 & -0.82863 & 1.18007 \\ \mathrm{O} & -0.18563 & -1.33352 & 1.45590 \\ \mathrm{C} & -1.24942 & -0.50680 & 1.18007 \\ \mathrm{O} & -1.06205 & 0.82752 & 1.45590 \\ \mathrm{C} & 0.36490 & 2.61717 & 0.83940 \\ \mathrm{C} & 2.08408 & -1.62460 & 0.83940 \\ \mathrm{C} & -2.44899 & -0.99257 & 0.83940 \\ \mathrm{~S} & -0.98869 & 3.75871 & 0.64646 \\ \mathrm{~S} & 3.74948 & -1.02313 & 0.64646 \\ \mathrm{~S} & -2.76080 & -2.73558 & 0.64646 \\ \mathrm{C} & 0.00000 & 4.94046 & -0.25383 \\ \mathrm{C} & 4.27856 & -2.47023 & -0.25383 \\ \mathrm{C} & -4.27856 & -2.47023 & -0.25383 \\ \mathrm{C} & 1.33573 & 4.75260 & -0.25651 \\ \mathrm{C} & 3.44801 & -3.53308 & -0.25651 \\ \mathrm{C} & -4.78374 & -1.21953 & -0.25651 \\ \mathrm{~S} & 1.97432 & 3.35042 & 0.64097 \\ \mathrm{~S} & 1.91439 & -3.38502 & 0.64097 \\ \mathrm{~S} & -3.88871 & 0.03460 & 0.64097 \\ \mathrm{~S} & 2.48406 & 5.91272 & -0.92414 \\ \mathrm{~S} & 3.87854 & -5.10762 & -0.92414 \\ \mathrm{~S} & -6.36260 & -0.80510 & -0.92414 \\ \mathrm{C} & 1.62118 & 7.42052 & -0.30809 \\ \mathrm{C} & 5.61577 & -5.11424 & -0.30809 \\ \mathrm{C} & -7.23695 & -2.30627 & -0.30809 \\ \mathrm{C} & 0.29546 & 7.67705 & -1.01026 \\ \mathrm{C} & 6.50079 & -4.09440 & -1.01026 \\ \mathrm{C} & -6.79625 & -3.58265 & -1.01026 \\ \mathrm{~S} & -0.90247 & 6.25024 & -1.01843 \\ \mathrm{~S} & 5.86410 & -2.34356 & -1.01843 \\ \mathrm{~S} & -4.96163 & -3.90668 & -1.01843 \\ \mathrm{H} & 1.49436 & 7.31827 & 0.77256 \\ & & & \end{array}$


Molecule 1e

$\begin{array}{rrrr}\mathrm{H} & 5.59063 & -4.95329 & 0.77256 \\ \mathrm{H} & -7.08499 & -2.36498 & 0.77256 \\ \mathrm{H} & 2.29748 & 8.25967 & -0.50160 \\ \mathrm{H} & 6.00435 & -6.11951 & -0.50160 \\ \mathrm{H} & -8.30183 & -2.14016 & -0.50160 \\ \mathrm{H} & 0.46574 & 7.93395 & -2.05811 \\ \mathrm{H} & 6.63813 & -4.37031 & -2.05811 \\ \mathrm{H} & -7.10387 & -3.56364 & -2.05811 \\ \mathrm{H} & -0.22368 & 8.50951 & -0.52266 \\ \mathrm{H} & 7.48129 & -4.06105 & -0.52266 \\ \mathrm{H} & -7.25761 & -4.44846 & -0.52266\end{array}$

$\begin{array}{rrrr}\mathrm{C} & 0.20064 & 1.30131 & -1.98813 \\ \mathrm{O} & -1.04515 & 0.83902 & -2.37147 \\ \mathrm{C} & -1.22729 & -0.47690 & -1.98813 \\ \mathrm{O} & -0.20403 & -1.32463 & -2.37147 \\ \mathrm{C} & 1.02665 & -0.82442 & -1.98813 \\ \mathrm{O} & 1.24918 & 0.48562 & -2.37147 \\ \mathrm{C} & 0.37428 & 2.43091 & -1.30883 \\ \mathrm{C} & -2.29237 & -0.89132 & -1.30883 \\ \mathrm{C} & 1.91809 & -1.53959 & -1.30883 \\ \mathrm{Se} & 2.10024 & 3.06261 & -0.79709 \\ \mathrm{Se} & -3.70243 & 0.28756 & -0.79709 \\ \mathrm{Se} & 1.60218 & -3.35017 & -0.79709 \\ \mathrm{C} & 1.33032 & 4.17220 & 0.57043 \\ \mathrm{C} & -4.27840 & -0.93401 & 0.57043 \\ \mathrm{C} & 2.94807 & -3.23819 & 0.57043 \\ \mathrm{C} & 0.00000 & 4.37738 & 0.56729 \\ \mathrm{C} & -3.79092 & -2.18869 & 0.56729 \\ \mathrm{C} & 3.79092 & -2.18869 & 0.56729 \\ \mathrm{Se} & -1.07398 & 3.55853 & -0.79442 \\ \mathrm{Se} & -2.54479 & -2.70936 & -0.79442 \\ \mathrm{Se} & 3.61877 & -0.84917 & -0.79442 \\ \mathrm{~S} & -0.82021 & 5.48791 & 1.66527 \\ \mathrm{~S} & -4.34256 & -3.45428 & 1.66527 \\ \mathrm{~S} & 5.16278 & -2.03363 & 1.66527 \\ \mathrm{C} & 0.46337 & 6.80878 & 1.62797 \\ \mathrm{C} & -6.12826 & -3.00310 & 1.62797 \\ \mathrm{C} & 5.66489 & -3.80568 & 1.62797 \\ \mathrm{C} & 1.75514 & 6.39765 & 2.31974 \\ \mathrm{C} & -6.41810 & -1.67883 & 2.31974 \\ \mathrm{C} & 4.66296 & -4.71882 & 2.31974 \\ \mathrm{~S} & 2.51392 & 4.81034 & 1.71305 \\ \mathrm{~S} & -5.42283 & -0.22805 & 1.71305 \\ \mathrm{~S} & 2.90891 & -4.58229 & 1.71305 \\ \mathrm{H} & 0.63350 & 7.08375 & 0.58387\end{array}$




$\begin{array}{rrrr}\mathrm{H} & -6.45145 & -2.99325 & 0.58387 \\ \mathrm{H} & 5.81796 & -4.09050 & 0.58387 \\ \mathrm{H} & 0.03230 & 7.66911 & 2.15023 \\ \mathrm{H} & -6.65779 & -3.80659 & 2.15023 \\ \mathrm{H} & 6.62549 & -3.86252 & 2.15023 \\ \mathrm{H} & 1.58575 & 6.26756 & 3.39093 \\ \mathrm{H} & -6.22074 & -1.76048 & 3.39093 \\ \mathrm{H} & 4.63499 & -4.50708 & 3.39093 \\ \mathrm{H} & 2.51485 & 7.17373 & 2.17440 \\ \mathrm{H} & -7.47005 & -1.40894 & 2.17440 \\ \mathrm{H} & 4.95520 & -5.76479 & 2.17440\end{array}$

Molecule 1f

$\begin{array}{lrrr}\mathrm{C} & -0.18246 & 1.33189 & -0.95788 \\ \mathrm{O} & -1.24704 & 0.50913 & -1.24929 \\ \mathrm{C} & -1.06222 & -0.82396 & -0.95788 \\ \mathrm{O} & 0.18260 & -1.33453 & -1.24929 \\ \mathrm{C} & 1.24468 & -0.50793 & -0.95788 \\ \mathrm{O} & 1.06444 & 0.82540 & -1.24929 \\ \mathrm{C} & -0.35331 & 2.59829 & -0.56178 \\ \mathrm{C} & -2.07353 & -1.60512 & -0.56178 \\ \mathrm{C} & 2.42684 & -0.99317 & -0.56178 \\ \mathrm{~S} & 1.03717 & 3.69560 & -0.32220 \\ \mathrm{~S} & -3.71907 & -0.94959 & -0.32220 \\ \mathrm{~S} & 2.68190 & -2.74601 & -0.32220 \\ \mathrm{C} & 0.00000 & 5.02598 & 0.22547 \\ \mathrm{C} & -4.35262 & -2.51299 & 0.22547 \\ \mathrm{C} & 4.35262 & -2.51299 & 0.22547 \\ \mathrm{C} & -1.32871 & 4.84719 & 0.23054 \\ \mathrm{C} & -3.53344 & -3.57429 & 0.23054 \\ \mathrm{C} & 4.86214 & -1.27290 & 0.23054 \\ \mathrm{~S} & -1.98215 & 3.29106 & -0.31408 \\ \mathrm{~S} & -1.85907 & -3.36213 & -0.31408 \\ \mathrm{~S} & 3.84122 & 0.07107 & -0.31408 \\ \mathrm{O} & -2.23811 & 5.79856 & 0.60625 \\ \mathrm{O} & -3.90265 & -4.83754 & 0.60625 \\ \mathrm{O} & 6.14076 & -0.96102 & 0.60625 \\ \mathrm{C} & -1.62300 & 7.09340 & 0.65319 \\ \mathrm{C} & -5.33157 & -4.95226 & 0.65319 \\ \mathrm{C} & 6.95457 & -2.14114 & 0.65319 \\ \mathrm{C} & -0.26903 & 7.01636 & 1.35837 \\ \mathrm{C} & -5.94183 & -3.74117 & 1.35837 \\ \mathrm{C} & 6.21086 & -3.27519 & 1.35837 \\ \mathrm{O} & 0.63114 & 6.17155 & 0.62848 \\ \mathrm{O} & -5.66029 & -2.53920 & 0.62848 \\ \mathrm{O} & 5.02915 & -3.63236 & 0.62848\end{array}$




$\begin{array}{lrrr}\mathrm{H} & -1.49190 & 7.47097 & -0.36899 \\ \mathrm{H} & -5.72410 & -5.02751 & -0.36899 \\ \mathrm{H} & 7.21600 & -2.44346 & -0.36899 \\ \mathrm{H} & -2.31485 & 7.74070 & 1.19704 \\ \mathrm{H} & -5.54622 & -5.87507 & 1.19704 \\ \mathrm{H} & 7.86107 & -1.86563 & 1.19704 \\ \mathrm{H} & -0.39838 & 6.61868 & 2.37315 \\ \mathrm{H} & -5.53276 & -3.65434 & 2.37315 \\ \mathrm{H} & 5.93113 & -2.96434 & 2.37315 \\ \mathrm{H} & 0.20672 & 7.99856 & 1.40824 \\ \mathrm{H} & -7.03031 & -3.82025 & 1.40824 \\ \mathrm{H} & 6.82359 & -4.17830 & 1.40824\end{array}$

Molecule 2a

$\begin{array}{lrrr}\text { C } & 0.22039 & 1.57492 & 1.92942 \\ \text { S } & 1.64528 & 0.66553 & 2.51271 \\ \text { C } & 1.25373 & -0.97833 & 1.92942 \\ \text { S } & -0.24628 & -1.75762 & 2.51271 \\ \text { C } & -1.47412 & -0.59660 & 1.92942 \\ \text { S } & -1.39901 & 1.09209 & 2.51271 \\ \text { C } & 0.38313 & 2.71781 & 1.22036 \\ \mathrm{C} & 2.16213 & -1.69070 & 1.22036 \\ \mathrm{C} & -2.54526 & -1.02710 & 1.22036 \\ \mathrm{~S} & -0.96179 & 3.78794 & 0.75495 \\ \mathrm{~S} & 3.76135 & -1.06104 & 0.75495 \\ \mathrm{~S} & -2.79956 & -2.72691 & 0.75495 \\ \mathrm{C} & 0.00000 & 4.84680 & -0.30801 \\ \mathrm{C} & 4.19746 & -2.42340 & -0.30801 \\ \mathrm{C} & -4.19746 & -2.42340 & -0.30801 \\ \mathrm{C} & 1.34033 & 4.65272 & -0.31326 \\ \mathrm{C} & 3.35921 & -3.48712 & -0.31326 \\ \mathrm{C} & -4.69954 & -1.16560 & -0.31326 \\ \mathrm{~S} & 1.97194 & 3.36559 & 0.74570 \\ \mathrm{~S} & 1.92871 & -3.39055 & 0.74570 \\ \mathrm{~S} & -3.90066 & 0.02496 & 0.74570 \\ \mathrm{~S} & -0.85824 & 6.14187 & -1.15480 \\ \mathrm{~S} & 5.74814 & -2.32768 & -1.15480 \\ \mathrm{~S} & -4.88989 & -3.81420 & -1.15480 \\ \mathrm{~S} & 2.52909 & 5.64142 & -1.17424 \\ \mathrm{~S} & 3.62107 & -5.01097 & -1.17424 \\ \mathrm{~S} & -6.15016 & -0.63046 & -1.17424 \\ \mathrm{C} & -2.01775 & 5.18894 & -2.21577 \\ \mathrm{C} & 5.50263 & -0.84705 & -2.21577 \\ \mathrm{C} & -3.48488 & -4.34189 & -2.21577 \\ \mathrm{C} & 3.27510 & 4.40590 & -2.31272 \\ \mathrm{C} & 2.17807 & -5.03927 & -2.31272\end{array}$




$\begin{array}{lrrr}\mathrm{C} & -5.45318 & 0.63337 & -2.31272 \\ \mathrm{H} & -2.60254 & 5.93734 & -2.75744 \\ \mathrm{H} & 6.44316 & -0.71481 & -2.75744 \\ \mathrm{H} & -3.84062 & -5.22254 & -2.75744 \\ \mathrm{H} & -1.47064 & 4.57049 & -2.93074 \\ \mathrm{H} & 4.69348 & -1.01163 & -2.93074 \\ \mathrm{H} & -3.22284 & -3.55886 & -2.93074 \\ \mathrm{H} & -2.69182 & 4.57032 & -1.61981 \\ \mathrm{H} & 5.30392 & 0.04603 & -1.61981 \\ \mathrm{H} & -2.61210 & -4.61635 & -1.61981 \\ \mathrm{H} & 4.05604 & 4.94101 & -2.85980 \\ \mathrm{H} & 2.25102 & -5.98314 & -2.85980 \\ \mathrm{H} & -6.30706 & 1.04213 & -2.85980 \\ \mathrm{H} & 3.72536 & 3.57672 & -1.76300 \\ \mathrm{H} & 1.23485 & -5.01462 & -1.76300 \\ \mathrm{H} & -4.96021 & 1.43790 & -1.76300 \\ \mathrm{H} & 2.53048 & 4.03101 & -3.01837 \\ \mathrm{H} & 2.22572 & -4.20696 & -3.01837 \\ \mathrm{H} & -4.75620 & 0.17595 & -3.01837\end{array}$

Molecule 2b

$\begin{array}{lrrr}\text { C } & -0.00005 & 1.58822 & 0.92052 \\ \text { S } & 1.53421 & 0.88574 & 1.51093 \\ \text { C } & 1.37546 & -0.79406 & 0.92052 \\ \text { S } & -0.00003 & -1.77154 & 1.51093 \\ \text { C } & -1.37541 & -0.79415 & 0.92052 \\ \text { S } & -1.53418 & 0.88579 & 1.51093 \\ \text { C } & 0.00000 & 2.71426 & 0.16552 \\ \text { C } & 2.35062 & -1.35713 & 0.16552 \\ \text { C } & -2.35062 & -1.35713 & 0.16552 \\ \text { S } & -1.49254 & 3.53664 & -0.36561 \\ \text { S } & 3.80909 & -0.47574 & -0.36561 \\ \text { S } & -2.31655 & -3.06090 & -0.36561 \\ \text { C } & -0.66840 & 4.79642 & -1.28004 \\ \text { C } & 4.48802 & -1.81936 & -1.28004 \\ \text { C } & -3.81962 & -2.97706 & -1.28004 \\ \text { C } & 0.66857 & 4.79552 & -1.28124 \\ \text { C } & 3.81876 & -2.97676 & -1.28124 \\ \text { C } & -4.48733 & -1.81876 & -1.28124 \\ \text { S } & 1.49267 & 3.53559 & -0.36697 \\ \text { S } & 2.31558 & -3.06049 & -0.36697 \\ \text { S } & -3.80825 & -0.47510 & -0.36697 \\ \text { H } & 1.29091 & 5.51397 & -1.80232 \\ \text { H } & 4.12978 & -3.87495 & -1.80232 \\ \text { H } & -5.42070 & -1.63902 & -1.80232 \\ \text { H } & -1.29073 & 5.51576 & -1.79989\end{array}$




$\begin{array}{lrrr}\mathrm{H} & 5.42215 & -1.64008 & -1.79989 \\ \mathrm{H} & -4.13142 & -3.87568 & -1.79989\end{array}$

Molecule 2c

Molecule 2d

$\begin{array}{crrr}\mathrm{C} & 0.70166 & 1.39326 & 1.42624 \\ \mathrm{~S} & 1.76614 & 0.10087 & 2.06514 \\ \mathrm{C} & 0.85577 & -1.30429 & 1.42624 \\ \mathrm{~S} & -0.79571 & -1.57996 & 2.06514 \\ \mathrm{C} & -1.55743 & -0.08898 & 1.42624 \\ \mathrm{~S} & -0.97043 & 1.47909 & 2.06514 \\ \mathrm{C} & 1.11856 & 2.22043 & 0.44404 \\ \mathrm{C} & 1.36366 & -2.07892 & 0.44404 \\ \mathrm{C} & -2.48223 & -0.14151 & 0.44404 \\ \mathrm{Se} & 0.00000 & 3.56661 & -0.31748 \\ \mathrm{Se} & 3.08878 & -1.78331 & -0.31748 \\ \mathrm{Se} & -3.08878 & -1.78331 & -0.31748 \\ \mathrm{C} & 1.34561 & 4.15384 & -1.52252 \\ \mathrm{C} & 2.92452 & -3.24225 & -1.52252 \\ \mathrm{C} & -4.27013 & -0.91159 & -1.52252 \\ \mathrm{C} & 2.53802 & 3.55252 & -1.52259 \\ \mathrm{C} & 1.80756 & -3.97425 & -1.52259 \\ \mathrm{C} & -4.34558 & 0.42173 & -1.52259 \\ \mathrm{Se} & 2.86553 & 2.12042 & -0.31851 \\ \mathrm{Se} & 0.40358 & -3.54183 & -0.31851 \\ \mathrm{Se} & -3.26910 & 1.42141 & -0.31851 \\ \mathrm{H} & 3.35291 & 3.82640 & -2.18473 \\ \mathrm{H} & 1.63731 & -4.81690 & -2.18473 \\ \mathrm{H} & -4.99021 & 0.99050 & -2.18473 \\ \mathrm{H} & 1.08115 & 4.97170 & -2.18483 \\ \mathrm{H} & 3.76504 & -3.42215 & -2.18483 \\ \mathrm{H} & -4.84619 & -1.54955 & -2.18483 \\ \mathrm{C} & -0.96576 & 4.66923 & -0.49933 \\ \mathrm{C} & -0.09548 & 1.58400 & 1.80999 \\ \mathrm{~S} & 1.47949 & 0.97745 & 2.39969 \\ \mathrm{C} & 1.41953 & -0.70931 & 1.80999 \\ \mathrm{~S} & 0.10674 & -1.77000 & 2.39969 \\ \mathrm{C} & -1.32404 & -0.87469 & 1.80999 \\ \mathrm{~S} & -1.58624 & 0.79256 & 2.39969 \\ \mathrm{C} & -0.16343 & 2.71978 & 1.07364 \\ \mathrm{C} & 2.43711 & -1.21835 & 1.07364 \\ \mathrm{~S} & -2.27368 & -1.50143 & 1.07364 \\ \mathrm{C} & 3.85840 & -0.25806 & 0.58712 \\ \mathrm{C} & -2.15269 & -3.21245 & 0.58712 \\ \mathrm{C} & -1.49824 & -0.49933\end{array}$




$\begin{array}{lrrr}\mathrm{C} & -3.56079 & -3.17099 & -0.49933 \\ \mathrm{C} & 0.37950 & 4.75010 & -0.49986 \\ \mathrm{C} & 3.92396 & -2.70371 & -0.49986 \\ \mathrm{C} & -4.30346 & -2.04639 & -0.49986 \\ \mathrm{~S} & 1.26869 & 3.65755 & 0.58398 \\ \mathrm{~S} & 2.53319 & -2.92750 & 0.58398 \\ \mathrm{~S} & -3.80188 & -0.73005 & 0.58398 \\ \mathrm{~S} & 1.29793 & 5.96967 & -1.38832 \\ \mathrm{~S} & 4.52092 & -4.10888 & -1.38832 \\ \mathrm{~S} & -5.81885 & -1.86079 & -1.38832 \\ \mathrm{C} & 0.13527 & 7.36302 & -1.07333 \\ \mathrm{C} & 6.30893 & -3.79866 & -1.07333 \\ \mathrm{C} & -6.44420 & -3.56436 & -1.07333 \\ \mathrm{C} & -1.19266 & 7.20062 & -1.79877 \\ \mathrm{C} & 6.83225 & -2.56743 & -1.79877 \\ \mathrm{C} & -5.63959 & -4.63318 & -1.79877 \\ \mathrm{~S} & -2.11381 & 5.62198 & -1.44796 \\ \mathrm{~S} & 5.92568 & -0.98038 & -1.44796 \\ \mathrm{~S} & -3.81188 & -4.64160 & -1.44796 \\ \mathrm{H} & 0.00000 & 7.45744 & 0.00709 \\ \mathrm{H} & 6.45833 & -3.72872 & 0.00709 \\ \mathrm{H} & -6.45833 & -3.72872 & 0.00709 \\ \mathrm{H} & 0.63664 & 8.26391 & -1.44199 \\ \mathrm{H} & 6.83844 & -4.68330 & -1.44199 \\ \mathrm{H} & -7.47507 & -3.58061 & -1.44199 \\ \mathrm{H} & -1.03914 & 7.23638 & -2.87943 \\ \mathrm{H} & 6.78646 & -2.71827 & -2.87943 \\ \mathrm{H} & -5.74732 & -4.51811 & -2.87943 \\ \mathrm{H} & -1.87127 & 8.01147 & -1.51182 \\ \mathrm{H} & 7.87378 & -2.38517 & -1.51182 \\ \mathrm{H} & -6.00250 & -5.62631 & -1.51182\end{array}$

Molecule 2e

$\begin{array}{rrrr}\mathrm{C} & 0.70579 & 1.38290 & -2.42148 \\ \mathrm{~S} & -0.96420 & 1.48565 & -3.06932 \\ \mathrm{C} & -1.55053 & -0.08022 & -2.42148 \\ \mathrm{~S} & -0.80451 & -1.57785 & -3.06932 \\ \mathrm{C} & 0.84473 & -1.30269 & -2.42148 \\ \mathrm{~S} & 1.76872 & 0.09220 & -3.06932 \\ \mathrm{C} & 1.10729 & 2.17798 & -1.40761 \\ \mathrm{C} & -2.43983 & -0.13005 & -1.40761 \\ \mathrm{C} & 1.33254 & -2.04793 & -1.40761 \\ \mathrm{Se} & 2.85617 & 2.08006 & -0.64991 \\ \mathrm{Se} & -3.22947 & 1.43349 & -0.64991 \\ \mathrm{Se} & 0.37330 & -3.51355 & -0.64991 \\ \mathrm{C} & 2.40568 & 3.24038 & 0.80955\end{array}$




$\begin{array}{rrrl}\mathrm{C} & -4.00909 & 0.46319 & 0.80955 \\ \mathrm{C} & 1.60341 & -3.70357 & 0.80955 \\ \mathrm{C} & 1.20430 & 3.84516 & 0.80672 \\ \mathrm{C} & -3.93215 & -0.87962 & 0.80672 \\ \mathrm{C} & 2.72785 & -2.96553 & 0.80672 \\ \mathrm{Se} & 0.00000 & 3.52958 & -0.64614 \\ \mathrm{Se} & -3.05670 & -1.76479 & -0.64614 \\ \mathrm{Se} & 3.05670 & -1.76479 & -0.64614 \\ \mathrm{~S} & 0.68476 & 5.01536 & 2.02438 \\ \mathrm{~S} & -4.68580 & -1.91466 & 2.02438 \\ \mathrm{~S} & 4.00105 & -3.10069 & 2.02438 \\ \mathrm{C} & 2.30937 & 5.85954 & 2.21444 \\ \mathrm{C} & -6.22919 & -0.92979 & 2.21444 \\ \mathrm{C} & 3.91982 & -4.92974 & 2.21444 \\ \mathrm{C} & 3.35497 & 4.97602 & 2.87937 \\ \mathrm{C} & -5.98684 & 0.41748 & 2.87937 \\ \mathrm{C} & 2.63187 & -5.39350 & 2.87937 \\ \mathrm{~S} & 3.67128 & 3.34811 & 2.03893 \\ \mathrm{~S} & -4.73519 & 1.50537 & 2.03893 \\ \mathrm{~S} & 1.06391 & -4.85348 & 2.03893 \\ \mathrm{H} & 2.63372 & 6.20027 & 1.22776 \\ \mathrm{H} & -6.68646 & -0.81927 & 1.22776 \\ \mathrm{H} & 4.05273 & -5.38101 & 1.22776 \\ \mathrm{H} & 2.12511 & 6.73609 & 2.84399 \\ \mathrm{H} & -6.89618 & -1.52764 & 2.84399 \\ \mathrm{H} & 4.77107 & -5.20845 & 2.84399 \\ \mathrm{H} & 3.05757 & 4.74677 & 3.90497 \\ \mathrm{H} & -5.63961 & 0.27455 & 3.90497 \\ \mathrm{H} & 2.58204 & -5.02132 & 3.90497 \\ \mathrm{H} & 4.32001 & 5.49457 & 2.89926 \\ \mathrm{H} & -6.91844 & 0.99396 & 2.89926 \\ \mathrm{H} & 2.59843 & -6.48852 & 2.89926\end{array}$

Molecule 2f

$\begin{array}{lrrr}\mathrm{C} & -0.63108 & 1.45309 & 1.62686 \\ \mathrm{~S} & 1.05383 & 1.42249 & 2.22369 \\ \mathrm{C} & 1.57396 & -0.18002 & 1.62686 \\ \mathrm{~S} & 0.70500 & -1.62389 & 2.22369 \\ \mathrm{C} & -0.94288 & -1.27308 & 1.62686 \\ \mathrm{~S} & -1.75883 & 0.20140 & 2.22369 \\ \mathrm{C} & -1.07195 & 2.47037 & 0.84594 \\ \mathrm{C} & 2.67538 & -0.30685 & 0.84594 \\ \mathrm{C} & -1.60343 & -2.16352 & 0.84594 \\ \mathrm{~S} & -2.77339 & 2.60211 & 0.31853 \\ \mathrm{~S} & 3.64019 & 1.10077 & 0.31853 \\ \mathrm{~S} & -0.86680 & -3.70288 & 0.31853\end{array}$




$\begin{array}{lrrr}\mathrm{C} & -2.50249 & 4.08450 & -0.61077 \\ \mathrm{C} & 4.78853 & 0.12497 & -0.61077 \\ \mathrm{C} & -2.28603 & -4.20947 & -0.61077 \\ \mathrm{C} & -1.26950 & 4.60989 & -0.62250 \\ \mathrm{C} & 4.62703 & -1.20553 & -0.62250 \\ \mathrm{C} & -3.35753 & -3.40436 & -0.62250 \\ \mathrm{~S} & 0.00000 & 3.79067 & 0.29975 \\ \mathrm{~S} & 3.28282 & -1.89534 & 0.29975 \\ \mathrm{~S} & -3.28282 & -1.89534 & 0.29975 \\ \mathrm{O} & -0.91880 & 5.75660 & -1.28152 \\ \mathrm{O} & 5.44476 & -2.08260 & -1.28152 \\ \mathrm{O} & -4.52596 & -3.67400 & -1.28152 \\ \mathrm{C} & -2.08838 & 6.49548 & -1.66184 \\ \mathrm{C} & 6.66944 & -1.43915 & -1.66184 \\ \mathrm{C} & -4.58106 & -5.05633 & -1.66184 \\ \mathrm{C} & -3.14203 & 5.56214 & -2.25871 \\ \mathrm{C} & 6.38797 & -0.05999 & -2.25871 \\ \mathrm{C} & -3.24594 & -5.50214 & -2.25871 \\ \mathrm{O} & -3.57508 & 4.60248 & -1.28405 \\ \mathrm{O} & 5.77341 & 0.79488 & -1.28405 \\ \mathrm{O} & -2.19832 & -5.39735 & -1.28405 \\ \mathrm{H} & -2.49716 & 7.00642 & -0.78070 \\ \mathrm{H} & 7.31632 & -1.34061 & -0.78070 \\ \mathrm{H} & -4.81916 & -5.66581 & -0.78070 \\ \mathrm{H} & -1.75750 & 7.23599 & -2.39364 \\ \mathrm{H} & 7.14530 & -2.09595 & -2.39364 \\ \mathrm{H} & -5.38780 & -5.14004 & -2.39364 \\ \mathrm{H} & -2.72643 & 5.03495 & -3.12702 \\ \mathrm{H} & 5.72361 & -0.15632 & -3.12702 \\ \mathrm{H} & -2.99718 & -4.87863 & -3.12702 \\ \mathrm{H} & -4.03433 & 6.11599 & -2.55947 \\ \mathrm{H} & 7.31377 & 0.43584 & -2.55947 \\ \mathrm{H} & -3.27944 & -6.55183 & -2.55947\end{array}$

Molecule 3a

$\begin{array}{lrrr}\mathrm{C} & 0.19871 & 1.40057 & -1.39757 \\ \mathrm{~N} & -1.06514 & 0.83438 & -1.46348 \\ \mathrm{C} & -1.31228 & -0.52819 & -1.39757 \\ \mathrm{~N} & -0.19002 & -1.33963 & -1.46348 \\ \mathrm{C} & 1.11357 & -0.87237 & -1.39757 \\ \mathrm{~N} & 1.25516 & 0.50525 & -1.46348 \\ \mathrm{C} & 0.38883 & 2.74786 & -1.33814 \\ \mathrm{C} & -2.57413 & -1.03719 & -1.33814 \\ \mathrm{C} & 2.18530 & -1.71067 & -1.33814 \\ \mathrm{~S} & 1.97228 & 3.51888 & -1.11451 \\ \mathrm{~S} & -4.03358 & -0.05139 & -1.11451\end{array}$




$\begin{array}{lrrr}\mathrm{S} & 2.06130 & -3.46748 & -1.11451 \\ \mathrm{C} & 1.34083 & 4.64242 & 0.14987 \\ \mathrm{C} & -4.69087 & -1.16002 & 0.14987 \\ \mathrm{C} & 3.35004 & -3.48240 & 0.14987 \\ \mathrm{C} & 0.00000 & 4.84685 & 0.13516 \\ \mathrm{C} & -4.19749 & -2.42342 & 0.13516 \\ \mathrm{C} & 4.19749 & -2.42342 & 0.13516 \\ \mathrm{~S} & -0.92199 & 3.92572 & -1.10457 \\ \mathrm{~S} & -2.93877 & -2.76133 & -1.10457 \\ \mathrm{~S} & 3.86077 & -1.16439 & -1.10457 \\ \mathrm{~S} & 2.50327 & 5.52063 & 1.14426 \\ \mathrm{~S} & -6.03264 & -0.59242 & 1.14426 \\ \mathrm{~S} & 3.52937 & -4.92821 & 1.14426 \\ \mathrm{~S} & -0.95808 & 5.93444 & 1.15247 \\ \mathrm{~S} & -4.66034 & -3.79694 & 1.15247 \\ \mathrm{~S} & 5.61841 & -2.13750 & 1.15247 \\ \mathrm{C} & 3.30232 & 4.15642 & 2.07815 \\ \mathrm{C} & -5.25073 & 0.78168 & 2.07815 \\ \mathrm{C} & 1.94841 & -4.93811 & 2.07815 \\ \mathrm{C} & -0.35241 & 7.59594 & 0.65703 \\ \mathrm{C} & -6.40207 & -4.10316 & 0.65703 \\ \mathrm{C} & 6.75448 & -3.49277 & 0.65703 \\ \mathrm{H} & 4.09884 & 4.62035 & 2.66598 \\ \mathrm{H} & -6.05076 & 1.23952 & 2.66598 \\ \mathrm{H} & 1.95192 & -5.85987 & 2.66598 \\ \mathrm{H} & 2.58538 & 3.67795 & 2.74857 \\ \mathrm{H} & -4.47789 & 0.40003 & 2.74857 \\ \mathrm{H} & 1.89251 & -4.07798 & 2.74857 \\ \mathrm{H} & 3.73597 & 3.41984 & 1.39886 \\ \mathrm{H} & -4.82965 & 1.52552 & 1.39886 \\ \mathrm{H} & 1.09369 & -4.94536 & 1.39886 \\ \mathrm{H} & -1.01433 & 8.31180 & 1.15292 \\ \mathrm{H} & -6.69107 & -5.03433 & 1.15292 \\ \mathrm{H} & 7.70539 & -3.27747 & 1.15292 \\ & 0.67330 & 7.75834 & 0.99234 \\ \mathrm{H} & -7.05557 & -3.29607 & 0.99234 \\ \mathrm{H} & -6.475297 & -4.46226 & 0.99234 \\ \mathrm{H} & -1.72504 & -4.23406 & -0.42461 \\ \mathrm{H} & -3.49094 & -0.42461 \\ \mathrm{H} & 1.44467 & -1.23916 \\ \mathrm{H} & -2.32038 & -1.23916 \\ \mathrm{H} & & & \\ \mathrm{H} & -87571 & -1.23916\end{array}$

Molecule 3b

$\begin{array}{lrrr}\mathrm{C} & 0.52016 & 1.31668 & -0.42456 \\ \mathrm{~N} & -0.84362 & 1.06269 & -0.49570\end{array}$ 


$\begin{array}{lrrr}\mathrm{C} & -1.40036 & -0.20786 & -0.42456 \\ \mathrm{~N} & -0.49850 & -1.26194 & -0.49570 \\ \mathrm{C} & 0.88019 & -1.10881 & -0.42456 \\ \mathrm{~N} & 1.34213 & 0.19925 & -0.49570 \\ \mathrm{C} & 1.01919 & 2.58034 & -0.35913 \\ \mathrm{C} & -2.74423 & -0.40753 & -0.35913 \\ \mathrm{C} & 1.72504 & -2.17281 & -0.35913 \\ \mathrm{~S} & 0.00000 & 4.03071 & -0.16461 \\ \mathrm{~S} & -3.49070 & -2.01536 & -0.16461 \\ \mathrm{~S} & 3.49070 & -2.01536 & -0.16461 \\ \mathrm{C} & 1.18634 & 4.82445 & 0.89886 \\ \mathrm{C} & -4.77127 & -1.38482 & 0.89886 \\ \mathrm{C} & 3.58492 & -3.43963 & 0.89886 \\ \mathrm{C} & 2.43041 & 4.33332 & 0.89873 \\ \mathrm{C} & -4.96797 & -0.06186 & 0.89873 \\ \mathrm{C} & 2.53756 & -4.27146 & 0.89873 \\ \mathrm{~S} & 2.75440 & 2.94335 & -0.16494 \\ \mathrm{~S} & -3.92621 & 0.91371 & -0.16494 \\ \mathrm{~S} & 1.17181 & -3.85706 & -0.16494 \\ \mathrm{H} & 0.86730 & 5.70470 & 1.44494 \\ \mathrm{H} & -5.37407 & -2.10124 & 1.44494 \\ \mathrm{H} & 4.50676 & -3.60346 & 1.44494 \\ \mathrm{H} & 3.26489 & 4.75830 & 1.44451 \\ \mathrm{H} & -5.75325 & 0.44833 & 1.44451 \\ \mathrm{H} & 2.48836 & -5.20663 & 1.44451 \\ \mathrm{H} & -1.45345 & 1.83056 & -0.23012 \\ \mathrm{H} & -0.85858 & -2.17401 & -0.23012 \\ \mathrm{H} & 2.31204 & 0.34345 & -0.23012\end{array}$

Molecule 3c

$\begin{array}{lrrr}\mathrm{C} & 0.53857 & 1.30738 & -0.50094 \\ \mathrm{~N} & -0.82499 & 1.07097 & -0.54325 \\ \mathrm{C} & -1.40151 & -0.18727 & -0.50094 \\ \mathrm{~N} & -0.51499 & -1.24995 & -0.54325 \\ \mathrm{C} & 0.86294 & -1.12011 & -0.50094 \\ \mathrm{~N} & 1.33998 & 0.17898 & -0.54325 \\ \mathrm{C} & 1.05764 & 2.56607 & -0.46544 \\ \mathrm{C} & -2.75110 & -0.36709 & -0.46544 \\ \mathrm{C} & 1.69346 & -2.19898 & -0.46544 \\ \mathrm{Se} & 2.90351 & 2.92077 & -0.14597 \\ \mathrm{Se} & -3.98122 & 1.05412 & -0.14597 \\ \mathrm{Se} & 1.07771 & -3.97490 & -0.14597 \\ \mathrm{C} & 2.41530 & 4.10078 & 1.30022 \\ \mathrm{C} & -4.75902 & 0.04132 & 1.30022 \\ \mathrm{C} & 2.34373 & -4.14210 & 1.30022 \\ \mathrm{C} & 1.18121 & 4.60989 & 1.30172\end{array}$




$\begin{array}{lrrr}\mathrm{C} & -4.58288 & -1.28198 & 1.30172 \\ \mathrm{C} & 3.40167 & -3.32790 & 1.30172 \\ \mathrm{Se} & 0.00000 & 4.11935 & -0.14267 \\ \mathrm{Se} & -3.56746 & -2.05967 & -0.14267 \\ \mathrm{Se} & 3.56746 & -2.05967 & -0.14267 \\ \mathrm{H} & 3.17889 & 4.35161 & 2.02876 \\ \mathrm{H} & -5.35805 & 0.57720 & 2.02876 \\ \mathrm{H} & 2.17916 & -4.92881 & 2.02876 \\ \mathrm{H} & 0.81821 & 5.32549 & 2.03170 \\ \mathrm{H} & -5.02112 & -1.95415 & 2.03170 \\ \mathrm{H} & 4.20291 & -3.37134 & 2.03170 \\ \mathrm{H} & -1.42241 & 1.84597 & -0.25747 \\ \mathrm{H} & -0.88745 & -2.15483 & -0.25747 \\ \mathrm{H} & 2.30986 & 0.30885 & -0.25747\end{array}$

\section{Molecule 3d}

$\begin{array}{lrrr}\mathrm{C} & 0.07890 & 1.41210 & -1.30731 \\ \mathrm{~N} & -1.13086 & 0.73994 & -1.35069 \\ \mathrm{C} & -1.26236 & -0.63772 & -1.30731 \\ \mathrm{~N} & -0.07537 & -1.34932 & -1.35069 \\ \mathrm{C} & 1.18346 & -0.77438 & -1.30731 \\ \mathrm{~N} & 1.20624 & 0.60938 & -1.35069 \\ \mathrm{C} & 0.15334 & 2.77332 & -1.29014 \\ \mathrm{C} & -2.47843 & -1.25386 & -1.29014 \\ \mathrm{C} & 2.32509 & -1.51945 & -1.29014 \\ \mathrm{~S} & 1.67154 & 3.67515 & -1.09118 \\ \mathrm{~S} & -4.01854 & -0.38998 & -1.09118 \\ \mathrm{~S} & 2.34700 & -3.28517 & -1.09118 \\ \mathrm{C} & 0.94294 & 4.73477 & 0.17104 \\ \mathrm{C} & -4.57190 & -1.55078 & 0.17104 \\ \mathrm{C} & 3.62896 & -3.18400 & 0.17104 \\ \mathrm{C} & -0.40559 & 4.81042 & 0.16923 \\ \mathrm{C} & -3.96315 & -2.75646 & 0.16923 \\ \mathrm{C} & 4.36874 & -2.05396 & 0.16923 \\ \mathrm{~S} & -1.25321 & 3.83907 & -1.08668 \\ \mathrm{~S} & -2.69813 & -3.00485 & -1.08668 \\ \mathrm{~S} & 3.95134 & -0.83422 & -1.08668 \\ \mathrm{~S} & -1.33917 & 5.95684 & 1.12580 \\ \mathrm{~S} & -4.48919 & -4.13817 & 1.12580 \\ \mathrm{~S} & 5.82836 & -1.81867 & 1.12580 \\ \mathrm{C} & -0.16270 & 7.36834 & 0.99437 \\ \mathrm{C} & -6.29982 & -3.82507 & 0.99437 \\ \mathrm{C} & 6.46252 & -3.54327 & 0.99437 \\ \mathrm{C} & 1.14740 & 7.11762 & 1.72768 \\ \mathrm{C} & -6.73774 & -2.56514 & 1.72768 \\ \mathrm{C} & 5.59034 & -4.55248 & 1.72768\end{array}$




$\begin{array}{lrrr}\mathrm{S} & 2.09053 & 5.61043 & 1.18018 \\ \mathrm{~S} & -5.90404 & -0.99476 & 1.18018 \\ \mathrm{~S} & 3.81351 & -4.61566 & 1.18018 \\ \mathrm{H} & 0.00000 & 7.58079 & -0.06542 \\ \mathrm{H} & -6.56516 & -3.79040 & -0.06542 \\ \mathrm{H} & 6.56516 & -3.79040 & -0.06542 \\ \mathrm{H} & -0.67039 & 8.22565 & 1.44850 \\ \mathrm{H} & -6.78843 & -4.69340 & 1.44850 \\ \mathrm{H} & 7.45882 & -3.53225 & 1.44850 \\ \mathrm{H} & 0.96581 & 7.00826 & 2.79906 \\ \mathrm{H} & -6.55224 & -2.66771 & 2.79906 \\ \mathrm{H} & 5.58643 & -4.34055 & 2.79906 \\ \mathrm{H} & 1.82764 & 7.96229 & 1.57085 \\ \mathrm{H} & -7.80936 & -2.39836 & 1.57085 \\ \mathrm{H} & 5.98172 & -5.56393 & 1.57085 \\ \mathrm{H} & -1.96187 & 1.28401 & -1.13747 \\ \mathrm{H} & -0.13104 & -2.34104 & -1.13747 \\ \mathrm{H} & 2.09292 & 1.05703 & -1.13747\end{array}$

Molecule 3e

$\begin{array}{cccc}\mathrm{C} & -0.22334 & 1.39303 & -1.54954 \\ \mathrm{~N} & -1.25868 & 0.48059 & -1.57005 \\ \mathrm{C} & -1.09473 & -0.88993 & -1.54954 \\ \mathrm{~N} & 0.21314 & -1.33034 & -1.57005 \\ \mathrm{C} & 1.31807 & -0.50310 & -1.54954 \\ \mathrm{~N} & 1.04554 & 0.84975 & -1.57005 \\ \mathrm{C} & -0.43980 & 2.74347 & -1.56819 \\ \mathrm{C} & -2.15602 & -1.75261 & -1.56819 \\ \mathrm{C} & 2.59581 & -0.99086 & -1.56819 \\ \mathrm{Se} & 0.94009 & 3.98646 & -1.14237 \\ \mathrm{Se} & -3.92242 & -1.17909 & -1.14237 \\ \mathrm{Se} & 2.98233 & -2.80737 & -1.14237 \\ \mathrm{C} & 0.00000 & 4.38103 & 0.52148 \\ \mathrm{C} & -3.79409 & -2.19052 & 0.52148 \\ \mathrm{C} & 3.79409 & -2.19052 & 0.52148 \\ \mathrm{C} & -1.33116 & 4.17674 & 0.52583 \\ \mathrm{C} & -2.95158 & -3.24119 & 0.52583 \\ \mathrm{C} & 4.28274 & -0.93555 & 0.52583 \\ \mathrm{Se} & -2.12872 & 3.50242 & -1.11731 \\ \mathrm{Se} & -1.96882 & -3.59473 & -1.11731 \\ \mathrm{Se} & 4.09754 & 0.09232 & -1.11731 \\ \mathrm{~S} & -2.41933 & 4.64747 & 1.82483 \\ \mathrm{~S} & -2.81517 & -4.41894 & 1.82483 \\ \mathrm{~S} & 5.23450 & -0.22854 & 1.82483 \\ \mathrm{C} & -1.52302 & 6.16280 & 2.36408 \\ \mathrm{C} & -4.57563 & -4.40037 & 2.36408\end{array}$




$\begin{array}{lrrr}\mathrm{C} & 6.09865 & -1.76242 & 2.36408 \\ \mathrm{C} & -0.15077 & 5.86178 & 2.95401 \\ \mathrm{C} & -5.00106 & -3.06146 & 2.95401 \\ \mathrm{C} & 5.15183 & -2.80032 & 2.95401 \\ \mathrm{~S} & 1.03027 & 5.01842 & 1.79452 \\ \mathrm{~S} & -4.86122 & -1.61697 & 1.79452 \\ \mathrm{~S} & 3.83095 & -3.40145 & 1.79452 \\ \mathrm{H} & -1.45333 & 6.84394 & 1.51175 \\ \mathrm{H} & -5.20036 & -4.68059 & 1.51175 \\ \mathrm{H} & 6.65369 & -2.16335 & 1.51175 \\ \mathrm{H} & -2.15384 & 6.62474 & 3.13049 \\ \mathrm{H} & -4.66027 & -5.17765 & 3.13049 \\ \mathrm{H} & 6.81411 & -1.44709 & 3.13049 \\ \mathrm{H} & -0.25002 & 5.23282 & 3.84137 \\ \mathrm{H} & -4.40675 & -2.83294 & 3.84137 \\ \mathrm{H} & 4.65677 & -2.39989 & 3.84137 \\ \mathrm{H} & 0.34514 & 6.79604 & 3.24094 \\ \mathrm{H} & -6.05811 & -3.09912 & 3.24094 \\ \mathrm{H} & 5.71297 & -3.69692 & 3.24094 \\ \mathrm{H} & -2.17858 & 0.83104 & -1.30062 \\ \mathrm{H} & 0.36959 & -2.30223 & -1.30062 \\ \mathrm{H} & 1.80899 & 1.47119 & -1.30062\end{array}$

Molecule 3f

$\begin{array}{lrrr}\mathrm{C} & -0.19573 & 1.40161 & -1.08300 \\ \mathrm{~N} & -1.25516 & 0.50830 & -1.13590 \\ \mathrm{C} & -1.11597 & -0.87032 & -1.08300 \\ \mathrm{~N} & 0.18738 & -1.34116 & -1.13590 \\ \mathrm{C} & 1.31170 & -0.53130 & -1.08300 \\ \mathrm{~N} & 1.06779 & 0.83285 & -1.13590 \\ \mathrm{C} & -0.38473 & 2.74924 & -1.04661 \\ \mathrm{C} & -2.18855 & -1.70781 & -1.04661 \\ \mathrm{C} & 2.57328 & -1.04143 & -1.04661 \\ \mathrm{~S} & 0.95750 & 3.91327 & -0.86679 \\ \mathrm{~S} & -3.86774 & -1.12741 & -0.86679 \\ \mathrm{~S} & 2.91024 & -2.78586 & -0.86679 \\ \mathrm{C} & 0.00000 & 4.87284 & 0.29852 \\ \mathrm{C} & -4.22000 & -2.43642 & 0.29852 \\ \mathrm{C} & 4.22000 & -2.43642 & 0.29852 \\ \mathrm{C} & -1.33116 & 4.68818 & 0.29943 \\ \mathrm{C} & -3.39450 & -3.49690 & 0.29943 \\ \mathrm{C} & 4.72566 & -1.19127 & 0.29943 \\ \mathrm{~S} & -1.99276 & 3.50097 & -0.86013 \\ \mathrm{~S} & -2.03555 & -3.47627 & -0.86013 \\ \mathrm{~S} & 4.02831 & -0.02471 & -0.86013 \\ \mathrm{O} & -2.21303 & 5.43957 & 1.02750\end{array}$









$\begin{array}{lrrr}\mathrm{S} & -1.96445 & -3.87689 & -0.63347 \\ \mathrm{~S} & 4.33971 & 0.23718 & -0.63347 \\ \mathrm{~S} & 0.00000 & 7.30055 & -0.15562 \\ \mathrm{~S} & -6.32246 & -3.65027 & -0.15562 \\ \mathrm{~S} & 6.32246 & -3.65027 & -0.15562 \\ \mathrm{~S} & -3.31602 & 6.50849 & -0.16744 \\ \mathrm{~S} & -3.97851 & -6.12601 & -0.16744 \\ \mathrm{~S} & 7.29453 & -0.38249 & -0.16744 \\ \mathrm{C} & 1.18998 & 7.11380 & 1.23210 \\ \mathrm{C} & -6.75572 & -2.52635 & 1.23210 \\ \mathrm{C} & 5.56575 & -4.58745 & 1.23210 \\ \mathrm{C} & -4.29390 & 5.81191 & 1.22369 \\ \mathrm{C} & -2.88631 & -6.62458 & 1.22369 \\ \mathrm{C} & 7.18021 & 0.81267 & 1.22369 \\ \mathrm{H} & 1.64985 & 8.09759 & 1.35916 \\ \mathrm{H} & -7.83764 & -2.61998 & 1.35916 \\ \mathrm{H} & 6.18779 & -5.47760 & 1.35916 \\ \mathrm{H} & 0.67143 & 6.83502 & 2.15191 \\ \mathrm{H} & -6.25501 & -2.83603 & 2.15191 \\ \mathrm{H} & 5.58358 & -3.99898 & 2.15191 \\ \mathrm{H} & 1.96600 & 6.38192 & 0.99978 \\ \mathrm{H} & -6.50990 & -1.48836 & 0.99978 \\ \mathrm{H} & 4.54390 & -4.89356 & 0.99978 \\ \mathrm{H} & -5.15775 & 6.47259 & 1.33621 \\ \mathrm{H} & -3.02656 & -7.70304 & 1.33621 \\ \mathrm{H} & 8.18431 & 1.23045 & 1.33621 \\ \mathrm{H} & -4.64137 & 4.80097 & 1.00218 \\ \mathrm{H} & -1.83707 & -6.42003 & 1.00218 \\ \mathrm{H} & 6.47844 & 1.61906 & 1.00218 \\ \mathrm{H} & -3.71104 & 5.81689 & 2.14721 \\ \mathrm{H} & -3.18205 & -6.12230 & 2.14721 \\ \mathrm{H} & 6.89309 & 0.30541 & 2.14721 \\ & & & \\ & & \end{array}$

Molecule 4b

$\begin{array}{rrrr}\mathrm{P} & -0.00019 & 1.62606 & 0.78975 \\ \mathrm{~N} & -1.40497 & 0.81104 & 0.61602 \\ \mathrm{P} & -1.40811 & -0.81319 & 0.78975 \\ \mathrm{~N} & 0.00010 & -1.62226 & 0.61602 \\ \mathrm{P} & 1.40830 & -0.81287 & 0.78975 \\ \mathrm{~N} & 1.40487 & 0.81122 & 0.61602 \\ \mathrm{C} & 0.00000 & 3.11450 & -0.10641 \\ \mathrm{C} & -2.69724 & -1.55725 & -0.10641 \\ \mathrm{C} & 2.69724 & -1.55725 & -0.10641 \\ \mathrm{~S} & 1.47183 & 4.04203 & -0.34124 \\ \mathrm{~S} & -4.23641 & -0.74638 & -0.34124 \\ \mathrm{~S} & 2.76459 & -3.29565 & -0.34124\end{array}$




$\begin{array}{lrrr}\mathrm{C} & 0.67089 & 5.61522 & -0.32606 \\ \mathrm{C} & -5.19837 & -2.22660 & -0.32606 \\ \mathrm{C} & 4.52748 & -3.38861 & -0.32606 \\ \mathrm{C} & -0.66928 & 5.61569 & -0.32567 \\ \mathrm{C} & -4.52869 & -3.38746 & -0.32567 \\ \mathrm{C} & 5.19797 & -2.22823 & -0.32567 \\ \mathrm{~S} & -1.47132 & 4.04310 & -0.34060 \\ \mathrm{~S} & -2.76577 & -3.29575 & -0.34060 \\ \mathrm{~S} & 4.23709 & -0.74735 & -0.34060 \\ \mathrm{H} & 1.29460 & 6.50157 & -0.35036 \\ \mathrm{H} & -6.27783 & -2.12963 & -0.35036 \\ \mathrm{H} & 4.98323 & -4.37194 & -0.35036 \\ \mathrm{H} & -1.29245 & 6.50244 & -0.34977 \\ \mathrm{H} & -4.98506 & -4.37051 & -0.34977 \\ \mathrm{H} & 6.27750 & -2.13193 & -0.34977\end{array}$

Molecule 4c

$\begin{array}{crrr}\mathrm{P} & 0.00024 & 1.61735 & 0.48662 \\ \mathrm{~N} & -1.40317 & 0.81040 & 0.30993 \\ \mathrm{P} & -1.40078 & -0.80847 & 0.48662 \\ \mathrm{~N} & -0.00024 & -1.62038 & 0.30993 \\ \mathrm{P} & 1.40054 & -0.80888 & 0.48662 \\ \mathrm{~N} & 1.40341 & 0.80998 & 0.30993 \\ \mathrm{C} & 0.00000 & 3.19905 & -0.12193 \\ \mathrm{C} & -2.77045 & -1.59952 & -0.12193 \\ \mathrm{C} & 2.77045 & -1.59952 & -0.12193 \\ \mathrm{Se} & 1.58615 & 4.22625 & -0.19085 \\ \mathrm{Se} & -4.45311 & -0.73948 & -0.19085 \\ \mathrm{Se} & 2.86696 & -3.48677 & -0.19085 \\ \mathrm{C} & 0.66551 & 5.83050 & 0.27803 \\ \mathrm{C} & -5.38212 & -2.33890 & 0.27803 \\ \mathrm{C} & 4.71661 & -3.49160 & 0.27803 \\ \mathrm{C} & -0.67034 & 5.82954 & 0.27690 \\ \mathrm{C} & -4.71336 & -3.49530 & 0.27690 \\ \mathrm{C} & 5.38370 & -2.33424 & 0.27690 \\ \mathrm{Se} & -1.58757 & 4.22374 & -0.19322 \\ \mathrm{Se} & -2.86408 & -3.48675 & -0.19322 \\ \mathrm{Se} & 4.45165 & -0.73699 & -0.19322 \\ \mathrm{H} & 1.27283 & 6.70251 & 0.49708 \\ \mathrm{H} & -6.44096 & -2.24895 & 0.49708 \\ \mathrm{H} & 5.16813 & -4.45356 & 0.49708 \\ \mathrm{H} & -1.27931 & 6.70064 & 0.49494 \\ \mathrm{H} & -5.16326 & -4.45824 & 0.49494 \\ \mathrm{H} & 6.44258 & -2.24240 & 0.49494\end{array}$

\section{Molecule 4d}




\begin{tabular}{|c|c|c|c|}
\hline $\mathrm{P}$ & -0.19286 & 1.61262 & -0.70930 \\
\hline $\mathrm{N}$ & 1.29567 & 0.96991 & -0.54517 \\
\hline $\mathrm{P}$ & 1.49300 & -0.63929 & -0.70930 \\
\hline $\mathrm{N}$ & 0.19213 & -1.60704 & -0.54517 \\
\hline $\mathrm{P}$ & -1.30014 & -0.97333 & -0.70930 \\
\hline $\mathrm{N}$ & -1.48780 & 0.63713 & -0.54517 \\
\hline $\mathrm{C}$ & -0.37115 & 3.12246 & 0.11372 \\
\hline $\mathrm{C}$ & 2.88970 & -1.23980 & 0.11372 \\
\hline $\mathrm{C}$ & -2.51855 & -1.88266 & 0.11372 \\
\hline $\mathrm{S}$ & -1.93676 & 3.88079 & 0.32175 \\
\hline $\mathrm{S}$ & 4.32924 & -0.26311 & 0.32175 \\
\hline $\mathrm{S}$ & -2.39248 & -3.61768 & 0.32175 \\
\hline $\mathrm{C}$ & -1.34328 & 5.53242 & -0.01977 \\
\hline $\mathrm{C}$ & 5.46285 & -1.60289 & -0.01977 \\
\hline $\mathrm{C}$ & -4.11957 & -3.92952 & -0.01977 \\
\hline $\mathrm{C}$ & 0.00000 & 5.69009 & -0.01967 \\
\hline $\mathrm{C}$ & 4.92776 & -2.84504 & -0.01967 \\
\hline $\mathrm{C}$ & -4.92776 & -2.84504 & -0.01967 \\
\hline $\mathrm{S}$ & 0.96927 & 4.22906 & 0.31854 \\
\hline $\mathrm{S}$ & 3.17784 & -2.95394 & 0.31854 \\
\hline $\mathrm{S}$ & -4.14711 & -1.27512 & 0.31854 \\
\hline $\mathrm{S}$ & 0.81510 & 7.24821 & -0.13027 \\
\hline $\mathrm{S}$ & 5.86959 & -4.33000 & -0.13027 \\
\hline $\mathrm{S}$ & -6.68469 & -2.91821 & -0.13027 \\
\hline $\mathrm{C}$ & -0.38587 & 8.18881 & 0.90350 \\
\hline $\mathrm{C}$ & 7.28465 & -3.76024 & 0.90350 \\
\hline $\mathrm{C}$ & -6.89879 & -4.42858 & 0.90350 \\
\hline $\mathrm{C}$ & -1.74651 & 8.34576 & 0.24037 \\
\hline $\mathrm{C}$ & 8.10089 & -2.66036 & 0.24037 \\
\hline $\mathrm{C}$ & -6.35438 & -5.68540 & 0.24037 \\
\hline $\mathrm{S}$ & -2.58382 & 6.76092 & -0.26294 \\
\hline $\mathrm{S}$ & 7.14703 & -1.14281 & -0.26294 \\
\hline $\mathrm{S}$ & -4.56322 & -5.61811 & -0.26294 \\
\hline $\mathrm{H}$ & -0.46190 & 7.69300 & 1.87455 \\
\hline $\mathrm{H}$ & 6.89328 & -3.44648 & 1.87455 \\
\hline $\mathrm{H}$ & -6.43138 & -4.24652 & 1.87455 \\
\hline $\mathrm{H}$ & 0.05690 & 9.17918 & 1.05161 \\
\hline $\mathrm{H}$ & 7.92095 & -4.63887 & 1.05161 \\
\hline $\mathrm{H}$ & -7.97786 & -4.54031 & 1.05161 \\
\hline $\mathrm{H}$ & -1.65925 & 8.95238 & -0.66354 \\
\hline $\mathrm{H}$ & 8.58261 & -3.03924 & -0.66354 \\
\hline $\mathrm{H}$ & -6.92337 & -5.91314 & -0.66354 \\
\hline $\mathrm{H}$ & -2.43851 & 8.84018 & 0.93099 \\
\hline $\mathrm{H}$ & 8.87507 & -2.30827 & 0.93099 \\
\hline $\mathrm{H}$ & -6.43656 & -6.53190 & 0.93099 \\
\hline
\end{tabular}


Molecule 4e

$\begin{array}{cccc}\mathrm{P} & 0.00000 & 1.61753 & -0.23242 \\ \mathrm{~N} & -1.39696 & 0.80709 & -0.36761 \\ \mathrm{P} & -1.40082 & -0.80876 & -0.23242 \\ \mathrm{~N} & -0.00048 & -1.61335 & -0.36761 \\ \mathrm{P} & 1.40082 & -0.80876 & -0.23242 \\ \mathrm{~N} & 1.39744 & 0.80626 & -0.36761 \\ \mathrm{C} & 0.00286 & 3.22971 & -0.71179 \\ \mathrm{C} & -2.79844 & -1.61238 & -0.71179 \\ \mathrm{C} & 2.79558 & -1.61733 & -0.71179 \\ \mathrm{Se} & 1.58418 & 4.26779 & -0.67550 \\ \mathrm{Se} & -4.48811 & -0.76195 & -0.67550 \\ \mathrm{Se} & 2.90392 & -3.50584 & -0.67550 \\ \mathrm{C} & 0.70775 & 5.64521 & 0.36007 \\ \mathrm{C} & -5.24277 & -2.20968 & 0.36007 \\ \mathrm{C} & 4.53502 & -3.43553 & 0.36007 \\ \mathrm{C} & -0.63942 & 5.65578 & 0.36501 \\ \mathrm{C} & -4.57834 & -3.38164 & 0.36501 \\ \mathrm{C} & 5.21775 & -2.27414 & 0.36501 \\ \mathrm{Se} & -1.55760 & 4.29679 & -0.65201 \\ \mathrm{Se} & -2.94233 & -3.49732 & -0.65201 \\ \mathrm{Se} & 4.49993 & -0.79947 & -0.65201 \\ \mathrm{~S} & -1.61471 & 6.93648 & 1.07684 \\ \mathrm{~S} & -5.19981 & -4.86662 & 1.07684 \\ \mathrm{~S} & 6.81452 & -2.06986 & 1.07684 \\ \mathrm{C} & -0.51894 & 8.34172 & 0.60775 \\ \mathrm{C} & -6.96467 & -4.62028 & 0.60775 \\ \mathrm{H} & 7.48361 & -3.72145 & 0.60775 \\ \mathrm{H} & 0.81296 & 8.32666 & 1.34442 \\ \mathrm{C} & -7.61757 & -3.45929 & 1.34442 \\ \mathrm{C} & 6.80462 & -4.86737 & 1.34442 \\ \mathrm{~S} & 1.80958 & 6.76720 & 1.14944 \\ \mathrm{~S} & -6.76536 & -1.81646 & 1.14944 \\ \mathrm{~S} & 4.95578 & -4.95074 & 1.14944 \\ \mathrm{H} & -0.38130 & 8.31669 & -0.47637 \\ \mathrm{H} & -7.01182 & -4.48856 & -0.47637 \\ \mathrm{H} & 7.39312 & -3.82813 & -0.47637 \\ \mathrm{H} & -1.06636 & 9.25301 & 0.86965 \\ \mathrm{H} & -7.48016 & -5.55000 & 0.86965 \\ \mathrm{H} & 0.65466 & 8.46809 & 2.41576 \\ \mathrm{H} & -7.66091 & -3.66709 & 2.41576 \\ \mathrm{H} & -4.86108 & 9.13628 & 0.97295 \\ \mathrm{H} & -3.31147 & 0.97295 \\ \mathrm{H} & -5.82481 & 0.97295\end{array}$


Molecule 4f

$\begin{array}{lrrr}\mathrm{P} & -0.00362 & 1.62676 & 0.89611 \\ \mathrm{~N} & -1.40593 & 0.80806 & 0.72058 \\ \mathrm{P} & -1.40701 & -0.81651 & 0.89611 \\ \mathrm{~N} & 0.00316 & -1.62160 & 0.72058 \\ \mathrm{P} & 1.41062 & -0.81025 & 0.89611 \\ \mathrm{~N} & 1.40277 & 0.81354 & 0.72058 \\ \mathrm{C} & 0.00000 & 3.10774 & -0.01726 \\ \mathrm{C} & -2.69138 & -1.55387 & -0.01726 \\ \mathrm{C} & 2.69138 & -1.55387 & -0.01726 \\ \mathrm{~S} & 1.49145 & 3.99844 & -0.27339 \\ \mathrm{~S} & -4.20848 & -0.70758 & -0.27339 \\ \mathrm{~S} & 2.71702 & -3.29086 & -0.27339 \\ \mathrm{C} & 0.69974 & 5.58206 & -0.20208 \\ \mathrm{C} & -5.18408 & -2.18503 & -0.20208 \\ \mathrm{C} & 4.48434 & -3.39703 & -0.20208 \\ \mathrm{C} & -0.64701 & 5.59620 & -0.19309 \\ \mathrm{C} & -4.52294 & -3.35843 & -0.19309 \\ \mathrm{C} & 5.16996 & -2.23777 & -0.19309 \\ \mathrm{~S} & -1.47352 & 4.03008 & -0.25493 \\ \mathrm{~S} & -2.75339 & -3.29114 & -0.25493 \\ \mathrm{~S} & 4.22691 & -0.73894 & -0.25493 \\ \mathrm{O} & -1.40627 & 6.73173 & -0.21517 \\ \mathrm{O} & -5.12671 & -4.58373 & -0.21517 \\ \mathrm{O} & 6.53298 & -2.14799 & -0.21517 \\ \mathrm{C} & -0.61494 & 7.85325 & -0.63695 \\ \mathrm{C} & -6.49365 & -4.45918 & -0.63695 \\ \mathrm{C} & 7.10858 & -3.39407 & -0.63695 \\ \mathrm{C} & 0.71847 & 7.87175 & 0.10928 \\ \mathrm{C} & -7.17637 & -3.31366 & 0.10928 \\ \mathrm{C} & 6.45790 & -4.55809 & 0.10928 \\ \mathrm{O} & 1.48800 & 6.69731 & -0.19216 \\ \mathrm{O} & -6.54404 & -2.06001 & -0.19216 \\ \mathrm{O} & 5.05604 & -4.63730 & -0.19216 \\ \mathrm{H} & -0.44198 & 7.79020 & -1.71879 \\ \mathrm{H} & -6.52552 & -4.27787 & -1.71879 \\ \mathrm{H} & 6.96750 & -3.51233 & -1.71879 \\ \mathrm{H} & -1.20398 & 8.74475 & -0.41069 \\ \mathrm{H} & -6.97119 & -5.41506 & -0.41069 \\ \mathrm{H} & 8.17517 & -3.32970 & -0.41069 \\ \mathrm{H} & 0.53928 & 7.91630 & 1.19096 \\ \mathrm{H} & -7.12536 & -3.49112 & 1.19096 \\ \mathrm{H} & 6.58607 & -4.42518 & 1.19096 \\ \mathrm{H} & 1.33096 & 8.72373 & -0.19480 \\ \mathrm{H} & -8.22045 & -3.20922 & -0.19480 \\ & & & \end{array}$


Molecule 4g

$\begin{array}{llll}\mathrm{H} & 6.88949 & -5.51451 & -0.19480\end{array}$

$\begin{array}{lrrr}\text { S } & 2.717081 & 10.975416 & 1.520054 \\ \mathrm{C} & 2.476039 & 9.999374 & -0.000048 \\ \mathrm{~S} & 2.718292 & 10.975188 & -1.520122 \\ \mathrm{C} & 3.103689 & 12.533097 & -0.671349 \\ \mathrm{C} & 3.103154 & 12.533201 & 0.671334 \\ \mathrm{C} & 2.154838 & 8.702156 & -0.000075 \\ \mathrm{~S} & 1.916990 & 7.744147 & 1.529649 \\ \mathrm{C} & 1.531037 & 6.184022 & 0.699027 \\ \mathrm{C} & 1.531588 & 6.183889 & -0.699213 \\ \mathrm{~S} & 1.918195 & 7.743862 & -1.529829 \\ \mathrm{C} & 1.244515 & 5.023273 & -1.430443 \\ \mathrm{C} & 0.962689 & 3.886310 & -0.697584 \\ \mathrm{C} & 0.962139 & 3.886442 & 0.697367 \\ \mathrm{C} & 1.243390 & 5.023547 & 1.430235 \\ \mathrm{O} & 0.653819 & 2.642314 & 1.221224 \\ \mathrm{P} & 0.388744 & 1.569874 & -0.000107 \\ \mathrm{O} & 0.654780 & 2.642080 & -1.221430 \\ \mathrm{~S} & 3.412154 & 13.948604 & -1.748025 \\ \mathrm{C} & 5.295277 & 13.935387 & -1.777104 \\ \mathrm{~S} & 3.490551 & 13.929165 & 1.747986 \\ \mathrm{C} & 1.818188 & 14.794923 & 1.778268 \\ \mathrm{~N} & 1.513280 & 0.436519 & -0.000099 \\ \mathrm{P} & 1.165165 & -1.121650 & -0.000083 \\ \mathrm{O} & 1.960719 & -1.888140 & -1.221400 \\ \mathrm{C} & 2.884308 & -2.776897 & -0.697531 \\ \mathrm{C} & 2.884692 & -2.776483 & 0.697420 \\ \mathrm{C} & 3.728836 & -3.588593 & 1.430293 \\ \mathrm{C} & 4.590025 & -4.417931 & 0.699077 \\ \mathrm{C} & 4.589640 & -4.418345 & -0.699163 \\ \mathrm{C} & 3.728045 & -3.589438 & -1.430398 \\ \mathrm{~S} & 5.747328 & -5.533130 & -1.529771 \\ \mathrm{C} & 6.458921 & -6.217207 & -0.000036 \\ \mathrm{~S} & 5.748191 & -5.532243 & 1.529700 \\ \mathrm{O} & 1.961385 & -1.887409 & 1.221264 \\ \mathrm{C} & 7.421757 & -7.143972 & -0.000026 \\ \mathrm{~S} & 8.145705 & -7.841653 & -1.520114 \\ \mathrm{C} & 9.302216 & -8.954363 & -0.671362 \\ \mathrm{C} & 9.302586 & -8.953965 & 0.671321 \\ \mathrm{~S} & 8.146532 & -7.840742 & 1.520057 \\ \mathrm{~S} & 10.373851 & -9.929232 & -1.748057 \\ \mathrm{C} & 9.420864 & -11.553468 & -1.777142 \\ \mathrm{~S} & 10.317850 & -9.987439 & 1.747954 \\ \mathrm{C} & 11.903787 & -8.971990 & 1.778231 \\ \mathrm{~N} & -0.378617 & -1.528849 & -0.000075 \\ & & & \end{array}$




\begin{tabular}{|c|c|c|c|}
\hline & & & \\
\hline & & & \\
\hline & & & \\
\hline & & & \\
\hline & 6 & & \\
\hline & & & \\
\hline & -6.1 & & \\
\hline & & & \\
\hline & & & \\
\hline & -8.6 & -2.4 & \\
\hline & & & \\
\hline & -2.6 & -0.7 & \\
\hline & -9.8 & & \\
\hline & 0.8 & -3. & \\
\hline & & & \\
\hline & -12 & -3.5 & \\
\hline & & & \\
\hline & & & \\
\hline & & -2 . & \\
\hline & & & \\
\hline & & & \\
\hline J & & & \\
\hline $\mathrm{H}$ & & & \\
\hline I & & & \\
\hline 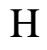 & & & \\
\hline & -4.9 & -1. & -2.5 \\
\hline$H$ & & & \\
\hline 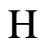 & & & -2.5 \\
\hline & -14 . & & \\
\hline & 12. & -9. & \\
\hline $\mathrm{H}$ & & 15. & \\
\hline & -15 . & & -2 \\
\hline & & 9 & \\
\hline & & & \\
\hline & -13 . & & \\
\hline & & & 0.7 \\
\hline H & & & \\
\hline & -15. & & -0 \\
\hline & & -11. & \\
\hline & & 14. & \\
\hline & -12. & & \\
\hline & & & \\
\hline & & 14. & 2.1 \\
\hline & -14.0 & & \\
\hline & & -11.3 & -2.14 \\
\hline & 5.650 & 12.972178 & -2.144 \\
\hline
\end{tabular}


Molecule 4h

$\begin{array}{rrrr}\mathrm{P} & 1.020227 & 1.255440 & -0.000022 \\ \mathrm{~N} & -0.561610 & 1.470788 & -0.000025 \\ \mathrm{P} & -1.597357 & 0.255823 & -0.000022 \\ \mathrm{~N} & -0.992935 & -1.221763 & -0.000025 \\ \mathrm{P} & 0.577129 & -1.511263 & -0.000022 \\ \mathrm{~N} & 1.554545 & -0.249026 & -0.000025 \\ \mathrm{O} & 1.716339 & 2.112104 & 1.221106 \\ \mathrm{O} & -2.687305 & 0.430342 & 1.221106 \\ \mathrm{O} & 0.970966 & -2.542445 & 1.221106 \\ \mathrm{C} & 2.525378 & 3.108104 & 0.697231 \\ \mathrm{C} & -3.954386 & 0.632990 & 0.697231 \\ \mathrm{C} & 1.429008 & -3.741093 & 0.697231 \\ \mathrm{C} & 2.525382 & 3.108108 & -0.697253 \\ \mathrm{C} & -3.954392 & 0.632991 & -0.697253 \\ \mathrm{C} & 1.429010 & -3.741100 & -0.697253 \\ \mathrm{O} & 1.716345 & 2.112111 & -1.221138 \\ \mathrm{O} & -2.687315 & 0.430343 & -1.221138 \\ \mathrm{O} & 0.970969 & -2.542454 & -1.221138 \\ \mathrm{C} & 3.255160 & 4.023581 & 1.430806 \\ \mathrm{C} & -5.112103 & 0.807261 & 1.430806 \\ \mathrm{C} & 1.856943 & -4.830842 & 1.430806 \\ \mathrm{C} & 4.009990 & 4.954376 & 0.702419 \\ \mathrm{C} & -6.295611 & 0.995565 & 0.702419 \\ \mathrm{C} & 2.285621 & -5.949942 & 0.702419 \\ \mathrm{C} & 4.009995 & 4.954381 & -0.702424 \\ \mathrm{C} & -6.295617 & 0.995567 & -0.702424 \\ \mathrm{C} & 2.285622 & -5.949947 & -0.702424 \\ \mathrm{C} & 3.255168 & 4.023589 & -1.430819 \\ \mathrm{C} & -5.112114 & 0.807264 & -1.430819 \\ \mathrm{C} & 1.856946 & -4.830852 & -1.430819 \\ \mathrm{~S} & 5.053350 & 6.156008 & 1.491066 \\ \mathrm{~S} & -7.857934 & 1.298325 & 1.491066 \\ \mathrm{~S} & 2.804585 & -7.454333 & 1.491066 \\ \mathrm{C} & 5.363297 & 7.080099 & 0.000008 \\ \mathrm{C} & -8.813194 & 1.104702 & 0.000008 \\ \mathrm{C} & 3.449897 & -8.184801 & 0.000008 \\ \mathrm{~S} & 5.053360 & 6.156016 & -1.491057 \\ \mathrm{~S} & -7.857946 & 1.298330 & -1.491057 \\ \mathrm{~S} & 2.804586 & -7.454346 & -1.491057 \\ \mathrm{C} & 5.822498 & 8.349595 & 0.000011 \\ \mathrm{C} & -10.142211 & 0.867634 & 0.000011 \\ \mathrm{C} & 4.319713 & -9.217229 & 0.000011 \\ \mathrm{~S} & 6.160936 & 9.266185 & -1.494620 \\ & & & \end{array}$




$\begin{array}{ccrc}\mathrm{S} & -11.105219 & 0.702435 & -1.494620 \\ \mathrm{~S} & 4.944283 & -9.968620 & -1.494620 \\ \mathrm{C} & 6.395927 & 10.806727 & -0.668455 \\ \mathrm{C} & -12.556863 & 0.135672 & -0.668455 \\ \mathrm{C} & 6.160936 & -10.942399 & -0.668455 \\ \mathrm{C} & 6.395923 & 10.806723 & 0.668496 \\ \mathrm{C} & -12.556858 & 0.135670 & 0.668496 \\ \mathrm{C} & 6.160935 & -10.942393 & 0.668496 \\ \mathrm{~S} & 6.160938 & 9.266173 & 1.494649 \\ \mathrm{~S} & -11.105210 & 0.702442 & 1.494649 \\ \mathrm{~S} & 4.944272 & -9.968615 & 1.494649 \\ \mathrm{H} & 6.553375 & 11.680607 & 1.289719 \\ \mathrm{H} & -13.392390 & -0.164914 & 1.289719 \\ \mathrm{H} & 6.839015 & -11.515693 & 1.289719 \\ \mathrm{H} & 6.553382 & 11.680616 & -1.289671 \\ \mathrm{H} & -13.392401 & -0.164913 & -1.289671 \\ \mathrm{H} & 6.839019 & -11.515703 & -1.289671 \\ \mathrm{H} & 3.235351 & 4.017039 & 2.514737 \\ \mathrm{H} & -5.096533 & 0.793377 & 2.514737 \\ \mathrm{H} & 1.861182 & -4.810416 & 2.514737 \\ \mathrm{H} & 3.235363 & 4.017052 & -2.514751 \\ \mathrm{H} & -5.096551 & 0.793380 & -2.514751 \\ \mathrm{H} & 1.861188 & -4.810433 & -2.514751\end{array}$

Molecule 4i

$\begin{array}{rrrr}\mathrm{P} & 0.194535 & 1.606137 & -0.000154 \\ \mathrm{~N} & -1.258989 & 0.945474 & -0.000173 \\ \mathrm{P} & -1.488223 & -0.634596 & -0.000154 \\ \mathrm{~N} & -0.189310 & -1.563054 & -0.000173 \\ \mathrm{P} & 1.293688 & -0.971541 & -0.000154 \\ \mathrm{~N} & 1.448300 & 0.617580 & -0.000173 \\ \mathrm{O} & 0.327910 & 2.701819 & 1.221267 \\ \mathrm{O} & -2.503799 & -1.066931 & 1.221267 \\ \mathrm{O} & 2.175889 & -1.634888 & 1.221267 \\ \mathrm{C} & 0.483555 & 3.975315 & 0.696819 \\ \mathrm{C} & -3.684502 & -1.568886 & 0.696819 \\ \mathrm{C} & 3.200946 & -2.406429 & 0.696819 \\ \mathrm{C} & 0.483542 & 3.975351 & -0.697010 \\ \mathrm{C} & -3.684526 & -1.568916 & -0.697010 \\ \mathrm{C} & 3.200984 & -2.406435 & -0.697010 \\ \mathrm{O} & 0.327866 & 2.701878 & -1.221515 \\ \mathrm{O} & -2.503828 & -1.066999 & -1.221515 \\ \mathrm{O} & 2.175962 & -1.634879 & -1.221515 \\ \mathrm{C} & 0.616085 & 5.140692 & 1.425670 \\ \mathrm{C} & -4.760012 & -2.036801 & 1.425670 \\ \mathrm{C} & 4.143927 & -3.103891 & 1.425670\end{array}$




$\begin{array}{cccc}\mathrm{C} & 0.766402 & 6.331851 & 0.701525 \\ \mathrm{C} & -5.866745 & -2.502202 & 0.701525 \\ \mathrm{C} & 5.100343 & -3.829649 & 0.701525 \\ \mathrm{C} & 0.766385 & 6.331885 & -0.701613 \\ \mathrm{C} & -5.866766 & -2.502233 & -0.701613 \\ \mathrm{C} & 5.100381 & -3.829651 & -0.701613 \\ \mathrm{C} & 0.616054 & 5.140759 & -1.425811 \\ \mathrm{C} & -4.760055 & -2.036861 & -1.425811 \\ \mathrm{C} & 4.144000 & -3.103898 & -1.425811 \\ \mathrm{Se} & 1.017432 & 8.009896 & 1.587682 \\ \mathrm{Se} & -7.445489 & -3.123826 & 1.587682 \\ \mathrm{Se} & 6.428057 & -4.886070 & 1.587682 \\ \mathrm{C} & 0.628217 & 8.991689 & 0.000018 \\ \mathrm{C} & -8.101140 & -3.951793 & 0.000018 \\ \mathrm{C} & 7.472923 & -5.039897 & 0.000018 \\ \mathrm{Se} & 1.017381 & 8.009970 & -1.587702 \\ \mathrm{Se} & -7.445529 & -3.123907 & -1.587702 \\ \mathrm{Se} & 6.428147 & -4.886063 & -1.587702 \\ \mathrm{C} & 0.164312 & 10.251795 & 0.000065 \\ \mathrm{C} & -8.960471 & -4.983599 & 0.000065 \\ \mathrm{C} & 8.796159 & -5.268196 & 0.000065 \\ \mathrm{Se} & -0.189310 & 11.236222 & -1.599873 \\ \mathrm{Se} & -9.636199 & -5.782058 & -1.599873 \\ \mathrm{Se} & 9.825509 & -5.454164 & -1.599873 \\ \mathrm{C} & -1.179344 & 12.564833 & -0.667374 \\ \mathrm{C} & -10.291792 & -7.303758 & -0.667374 \\ \mathrm{C} & 11.471136 & -5.261074 & -0.667374 \\ \mathrm{C} & -1.179340 & 12.564793 & 0.667643 \\ \mathrm{C} & -10.291760 & -7.303735 & 0.667643 \\ \mathrm{C} & 11.471100 & -5.261058 & 0.667643 \\ \mathrm{Se} & -0.189310 & 11.236124 & 1.600062 \\ \mathrm{Se} & -9.636113 & -5.782009 & 1.600062 \\ \mathrm{H} & -4.735337 & -2.044263 & -2.509859 \\ \mathrm{Se} & 9.825423 & -5.454115 & 1.600062 \\ \mathrm{H} & -1.700725 & 13.297304 & 1.274319 \\ \mathrm{H} & -10.665440 & -8.121523 & 1.274319 \\ \mathrm{H} & 12.366165 & -5.175781 & 1.274319 \\ \mathrm{H} & -1.700720 & 13.297391 & -1.274002 \\ \mathrm{H} & -10.665518 & -8.121562 & -1.274002 \\ \mathrm{H} & 12.366238 & -5.175829 & -1.274002 \\ \mathrm{H} & 0.597341 & 5.122938 & 2.509718 \\ \mathrm{H} & 4.137924 & -3.078782 & 2.509718 \\ \mathrm{H} & 0.597285 & 5.123054 & -2.509859 \\ \mathrm{H} & -3.078791 & -2.509859\end{array}$




\section{Molecule 4j}

$\begin{array}{rrrr}\mathrm{P} & 0.000376 & 1.616300 & -0.010615 \\ \mathrm{~N} & 1.363528 & 0.786900 & -0.010615 \\ \mathrm{P} & 1.399569 & -0.808476 & -0.010615 \\ \mathrm{~N} & -0.000289 & -1.574300 & -0.010615 \\ \mathrm{P} & -1.399945 & -0.807824 & -0.010615 \\ \mathrm{~N} & -1.363239 & 0.787400 & -0.010615 \\ \mathrm{O} & -0.000433 & 2.723892 & -1.229974 \\ \mathrm{O} & 2.359176 & -1.361571 & -1.229974 \\ \mathrm{O} & -2.358743 & -1.362321 & -1.229974 \\ \mathrm{C} & 0.000064 & 4.003810 & -0.707104 \\ \mathrm{C} & 3.467369 & -2.001960 & -0.707104 \\ \mathrm{C} & -3.467433 & -2.001850 & -0.707104 \\ \mathrm{C} & 0.001099 & 4.002322 & 0.690595 \\ \mathrm{C} & 3.465563 & -2.002112 & 0.690595 \\ \mathrm{C} & -3.466662 & -2.000209 & 0.690595 \\ \mathrm{O} & 0.001375 & 2.721561 & 1.210866 \\ \mathrm{O} & 2.356254 & -1.361971 & 1.210866 \\ \mathrm{O} & -2.357628 & -1.359590 & 1.210866 \\ \mathrm{C} & -0.000589 & 5.173007 & -1.440164 \\ \mathrm{C} & 4.480250 & -2.585994 & -1.440164 \\ \mathrm{C} & -4.479661 & -2.587014 & -1.440164 \\ \mathrm{C} & -0.000159 & 6.370872 & -0.708006 \\ \mathrm{C} & 5.517416 & -3.185298 & -0.708006 \\ \mathrm{C} & -5.517257 & -3.185574 & -0.708006 \\ \mathrm{C} & 0.000881 & 6.369199 & 0.696793 \\ \mathrm{C} & 5.515448 & -3.185363 & 0.696793 \\ \mathrm{C} & -5.516329 & -3.183836 & 0.696793 \\ \mathrm{C} & 0.001534 & 5.169784 & 1.426215 \\ \mathrm{C} & 4.476397 & -2.586220 & 1.426215 \\ \mathrm{C} & -4.477931 & -2.583563 & 1.426215 \\ \mathrm{~S} & -0.000893 & 7.951999 & -1.498225 \\ \mathrm{~S} & 6.887079 & -3.975226 & -1.498225 \\ \mathrm{~S} & -6.886186 & -3.976772 & -1.498225 \\ \mathrm{C} & 0.000126 & 8.902759 & -0.002294 \\ \mathrm{C} & 7.709953 & -4.451489 & -0.002294 \\ \mathrm{C} & -7.710079 & -4.451271 & -0.002294 \\ \mathrm{~S} & 0.001321 & 7.948030 & 1.491107 \\ \mathrm{~S} & 6.882535 & -3.975159 & 1.491107 \\ \mathrm{~S} & -6.883857 & -3.972871 & 1.491107 \\ \mathrm{C} & 0.000000 & 10.264357 & 0.000363 \\ \mathrm{C} & 8.889194 & -5.132178 & 0.000363 \\ \mathrm{C} & -8.889194 & -5.132178 & 0.000363 \\ \mathrm{~S} & 0.001012 & 11.221097 & 1.488079 \\ \mathrm{~S} & 9.717249 & -5.611425 & 1.488079 \\ \mathrm{~S} & -9.718261 & -5.609672 & 1.488079\end{array}$




$\begin{array}{lccc}\text { C } & 0.000268 & 12.801689 & 0.684139 \\ \text { C } & 11.086454 & -6.401077 & 0.684139 \\ \text { C } & -11.086722 & -6.400612 & 0.684139 \\ \text { C } & -0.000735 & 12.805291 & -0.669956 \\ \text { C } & 11.090075 & -6.402009 & -0.669956 \\ \text { C } & -11.089340 & -6.403282 & -0.669956 \\ \text { S } & -0.001188 & 11.228836 & -1.482198 \\ \text { S } & 9.725051 & -5.613389 & -1.482198 \\ \text { S } & -9.723863 & -5.615447 & -1.482198 \\ \text { S } & 0.016867 & 14.187756 & -1.776719 \\ \text { S } & 12.278523 & -7.108485 & -1.776719 \\ \text { S } & -12.295391 & -7.079270 & -1.776719 \\ \text { S } & -0.019165 & 14.177527 & 1.798779 \\ \text { S } & 12.287681 & -7.072167 & 1.798779 \\ \text { S } & -12.268516 & -7.105361 & 1.798779 \\ \text { C } & -0.421191 & 15.545849 & -0.623385 \\ \text { C } & 13.673695 & -7.408163 & -0.623385 \\ \text { C } & -13.252505 & -8.137686 & -0.623385 \\ \text { C } & 0.416507 & 15.542674 & 0.653214 \\ \text { C } & 13.252097 & -8.132043 & 0.653214 \\ \text { C } & -13.668605 & -7.410631 & 0.653214 \\ \text { H } & -0.218759 & 16.453382 & -1.201305 \\ \text { H } & 14.358426 & -8.037240 & -1.201305 \\ \text { H } & -14.139667 & -8.416142 & -1.201305 \\ \text { H } & -1.489309 & 15.509522 & -0.397722 \\ \text { H } & 14.176294 & -6.464982 & -0.397722 \\ \text { H } & -12.686986 & -9.044540 & -0.397722 \\ \text { H } & 1.484675 & 15.509165 & 0.427350 \\ \text { H } & 12.688993 & -9.040348 & 0.427350 \\ \text { H } & -14.173668 & -6.468816 & 0.427350 \\ \text { H } & 0.212827 & 16.446669 & 1.236408 \\ \text { H } & 14.136819 & -8.407648 & 1.236408 \\ \text { H } & -14.349646 & -8.039021 & 1.236408 \\ \text { H } & -0.001390 & 5.156645 & -2.524040 \\ \text { H } & 4.466480 & -2.577119 & -2.524040 \\ \text { H } & -4.465091 & -2.579526 & -2.524040 \\ \text { H } & 0.002338 & 5.150956 & 2.510051 \\ \text { H } & 4.459689 & -2.577503 & 2.510051 \\ \text { H } & -4.462028 & -2.573453 & 2.510051\end{array}$

Molecule 4k

$$
\begin{array}{crrr}
P & -0.059947 & 1.615735 & -0.019504 \\
N & -1.392569 & 0.736357 & -0.020571 \\
P & -1.369294 & -0.859783 & -0.019504 \\
N & 0.058581 & -1.574178 & -0.020571
\end{array}
$$




$\begin{array}{lrrr}\mathrm{P} & 1.429241 & -0.755952 & -0.019504 \\ \mathrm{~N} & 1.333988 & 0.837822 & -0.020571 \\ \mathrm{O} & -0.102193 & 2.718032 & 1.203208 \\ \mathrm{O} & -2.302788 & -1.447517 & 1.203208 \\ \mathrm{O} & 2.404981 & -1.270515 & 1.203208 \\ \mathrm{C} & -0.158170 & 3.999605 & 0.679529 \\ \mathrm{C} & -3.384675 & -2.136782 & 0.679529 \\ \mathrm{C} & 3.542845 & -1.862824 & 0.679529 \\ \mathrm{C} & -0.157840 & 4.000754 & -0.714430 \\ \mathrm{C} & -3.385835 & -2.137071 & -0.714430 \\ \mathrm{C} & 3.543675 & -1.863684 & -0.714430 \\ \mathrm{O} & -0.101444 & 2.720059 & -1.240200 \\ \mathrm{O} & -2.304919 & -1.447882 & -1.240200 \\ \mathrm{O} & 2.406362 & -1.272177 & -1.240200 \\ \mathrm{C} & -0.199659 & 5.170645 & 1.408903 \\ \mathrm{C} & -4.378081 & -2.758232 & 1.408903 \\ \mathrm{C} & 4.577740 & -2.412413 & 1.408903 \\ \mathrm{C} & -0.256903 & 6.371412 & 0.685421 \\ \mathrm{C} & -5.389353 & -3.408190 & 0.685421 \\ \mathrm{C} & 5.646256 & -2.963222 & 0.685421 \\ \mathrm{C} & -0.256441 & 6.372451 & -0.716673 \\ \mathrm{C} & -5.390484 & -3.408310 & -0.716673 \\ \mathrm{C} & 5.646925 & -2.964141 & -0.716673 \\ \mathrm{C} & -0.198741 & 5.172869 & -1.442031 \\ \mathrm{C} & -4.380466 & -2.758549 & -1.442031 \\ \mathrm{C} & 4.579207 & -2.414320 & -1.442031 \\ \mathrm{Se} & -0.364044 & 8.056860 & 1.580530 \\ \mathrm{Se} & -6.795423 & -4.343701 & 1.580530 \\ \mathrm{Se} & 7.159467 & -3.713159 & 1.580530 \\ \mathrm{C} & 0.000000 & 9.035837 & -0.013481 \\ \mathrm{C} & -7.825264 & -4.517918 & -0.013481 \\ \mathrm{C} & 7.825264 & -4.517918 & -0.013481 \\ \mathrm{Se} & -0.363849 & 8.059253 & -1.608626 \\ \mathrm{Se} & -6.797593 & -4.344729 & -1.608626 \\ \mathrm{Se} & 7.161443 & -3.714524 & -1.608626 \\ \mathrm{C} & 0.419610 & 10.311305 & -0.015272 \\ \mathrm{C} & -9.139657 & -4.792260 & -0.015272 \\ \mathrm{C} & 8.720047 & -5.519045 & -0.015272 \\ \mathrm{Se} & 0.747348 & 11.334112 & -1.591897 \\ \mathrm{Se} & -10.189303 & -5.019834 & -1.591897 \\ \mathrm{Se} & 9.441955 & -6.314278 & -1.591897 \\ \mathrm{C} & 2.073110 & 12.382127 & -0.673891 \\ \mathrm{C} & -11.759791 & -4.395698 & -0.673891 \\ \mathrm{C} & 9.686682 & -7.986429 & -0.673891 \\ \mathrm{C} & 2.068222 & 12.383039 & 0.672326 \\ \mathrm{C} & -11.758137 & -4.400387 & 0.672326 \\ & & & \end{array}$




$\begin{array}{cccc}\mathrm{C} & 9.689915 & -7.982652 & 0.672326 \\ \mathrm{Se} & 0.721603 & 11.334525 & 1.567736 \\ \mathrm{Se} & -10.176788 & -5.042336 & 1.567736 \\ \mathrm{Se} & 9.455185 & -6.292189 & 1.567736 \\ \mathrm{~S} & 3.134956 & 13.265458 & 1.761890 \\ \mathrm{~S} & -13.055702 & -3.917777 & 1.761890 \\ \mathrm{~S} & 9.920746 & -9.347681 & 1.761890 \\ \mathrm{~S} & 3.101920 & 13.425242 & -1.652424 \\ \mathrm{~S} & -13.177561 & -4.026279 & -1.652424 \\ \mathrm{~S} & 10.075640 & -9.398963 & -1.652424 \\ \mathrm{C} & 3.691237 & 14.733872 & 0.762782 \\ \mathrm{C} & -14.605526 & -4.170231 & 0.762782 \\ \mathrm{C} & 10.914289 & -10.563641 & 0.762782 \\ \mathrm{C} & 2.985656 & 14.914724 & -0.573999 \\ \mathrm{C} & -14.409358 & -4.871708 & -0.573999 \\ \mathrm{C} & 11.423702 & -10.043016 & -0.573999 \\ \mathrm{H} & 3.515100 & 15.611041 & 1.395498 \\ \mathrm{H} & -15.277108 & -4.761354 & 1.395498 \\ \mathrm{H} & 11.762008 & -10.849686 & 1.395498 \\ \mathrm{H} & 4.766928 & 14.621245 & 0.610852 \\ \mathrm{H} & -15.045834 & -3.182342 & 0.610852 \\ \mathrm{H} & 10.278906 & -11.438904 & 0.610852 \\ \mathrm{H} & 1.927483 & 15.155126 & -0.443052 \\ \mathrm{H} & -14.088466 & -5.908314 & -0.443052 \\ \mathrm{H} & 12.160983 & -9.246812 & -0.443052 \\ \mathrm{H} & 3.463906 & 15.729362 & -1.127446 \\ \mathrm{H} & -15.353979 & -4.864851 & -1.127446 \\ \mathrm{H} & 11.890074 & -10.864511 & -1.127446 \\ \mathrm{H} & -0.183470 & 5.151014 & 2.492976 \\ \mathrm{H} & -4.369174 & -2.734396 & 2.492976 \\ \mathrm{H} & 4.552643 & -2.416617 & 2.492976 \\ \mathrm{H} & -0.181899 & 5.154912 & -2.526115 \\ \mathrm{H} & -4.373335 & -2.734985 & -2.526115 \\ \mathrm{H} & 4.555234 & -2.419927 & -2.526115\end{array}$

Molecule 41

$\begin{array}{rrrr}\mathrm{P} & -0.000729 & -1.381722 & -0.840664 \\ \mathrm{~N} & -0.000729 & -1.381722 & 0.755975 \\ \mathrm{P} & -0.000729 & -0.037176 & 1.616938 \\ \mathrm{~N} & -0.000729 & 1.345555 & 0.818618 \\ \mathrm{P} & -0.000729 & 1.418897 & -0.776274 \\ \mathrm{~N} & -0.000729 & 0.036168 & -1.574594 \\ \mathrm{O} & -1.222017 & -2.325569 & -1.414643 \\ \mathrm{O} & -1.222017 & -0.062333 & 2.721323 \\ \mathrm{O} & -1.222017 & 2.387901 & -1.306680 \\ \mathrm{C} & -0.698292 & -3.421025 & -2.080466\end{array}$




$\begin{array}{rrrr}\text { C } & -0.698292 & -0.091224 & 4.002928 \\ \text { C } & -0.698292 & 3.512249 & -1.922462 \\ \text { C } & 0.696627 & -3.421065 & -2.080531 \\ \text { C } & 0.696627 & -0.091260 & 4.002995 \\ \text { C } & 0.696627 & 3.512325 & -1.922465 \\ \text { O } & 1.220478 & -2.325624 & -1.414748 \\ \text { O } & 1.220478 & -0.062395 & 2.721423 \\ \text { O } & 1.220478 & 2.388019 & -1.306677 \\ \text { C } & -1.431944 & -4.416006 & -2.699011 \\ \text { C } & -1.431944 & -0.129409 & 5.173879 \\ \text { C } & -1.431944 & 4.545416 & -2.474868 \\ \text { C } & -0.700223 & -5.436164 & -3.319998 \\ \text { C } & -0.700223 & -0.157120 & 6.367856 \\ \text { C } & -0.700223 & 5.593285 & -3.047857 \\ \text { C } & 0.698290 & -5.436156 & -3.320117 \\ \text { C } & 0.698290 & -0.157227 & 6.367908 \\ \text { C } & 0.698290 & 5.593385 & -3.047791 \\ \text { C } & 1.430140 & -4.416114 & -2.699121 \\ \text { C } & 1.430140 & -0.129450 & 5.174026 \\ \text { C } & 1.430140 & 4.545564 & -2.474905 \\ \text { S } & -1.520458 & -6.840939 & -4.123358 \\ \text { S } & -1.520458 & -0.150464 & 7.986106 \\ \text { S } & -1.520458 & 6.991402 & -3.862747 \\ \text { C } & -0.001033 & -7.464459 & -4.899823 \\ \text { C } & -0.001033 & -0.511141 & 8.914322 \\ \text { C } & -0.001033 & 7.975600 & -4.014499 \\ \text { S } & 1.518263 & -6.841575 & -4.123030 \\ \text { S } & 1.518263 & -0.149861 & 7.986493 \\ \text { S } & 1.518263 & 6.991436 & -3.863463 \\ \text { C } & -0.001494 & -8.336452 & -5.915046 \\ \text { C } & -0.001494 & -0.954354 & 10.177102 \\ \text { C } & -0.001494 & 9.290806 & -4.262056 \\ \text { S } & 1.535593 & -9.004122 & -6.636727 \\ \text { S } & 1.535593 & -1.245512 & 11.116162 \\ \text { S } & 1.535593 & 10.249635 & -4.479436 \\ \text { C } & 0.669637 & -9.700706 & -8.066391 \\ \text { C } & 0.669637 & -2.135346 & 12.434253 \\ \text { C } & 0.669637 & 11.836053 & -4.367862 \\ \text { C } & -0.667248 & -9.694471 & -8.071067 \\ \text { C } & -0.667248 & -2.142514 & 12.431191 \\ \text { C } & -0.667248 & 11.836984 & -4.360124 \\ \text { S } & -1.537281 & -8.995066 & -6.645899 \\ \text { S } & -1.537281 & -1.257984 & 11.112905 \\ \text { S } & -1.537281 & 10.253050 & -4.467006 \\ \text { O } & -1.442207 & -10.239986 & -9.052189 \\ \text { O } & -1.442207 & -2.719434 & 13.394183\end{array}$









\begin{tabular}{rrrr}
$\mathrm{C}$ & 4.530284 & -2.105651 & -0.468196 \\
$\mathrm{C}$ & -0.898450 & 3.707155 & -1.161433 \\
$\mathrm{C}$ & -2.761266 & -2.631658 & -1.161433 \\
$\mathrm{C}$ & 3.659716 & -1.075497 & -1.161433 \\
$\mathrm{O}$ & 0.076817 & 2.645582 & -1.110990 \\
$\mathrm{O}$ & -2.329549 & -1.256265 & -1.110990 \\
$\mathrm{O}$ & 2.252732 & -1.389316 & -1.110990 \\
$\mathrm{H}$ & 0.971052 & 3.934876 & 2.483338 \\
$\mathrm{H}$ & -3.893228 & -1.126482 & 2.483338 \\
$\mathrm{H}$ & 2.922176 & -2.808394 & 2.483338 \\
$\mathrm{H}$ & 1.755443 & 3.422305 & 0.986992 \\
$\mathrm{H}$ & -3.841524 & -0.190894 & 0.986992 \\
$\mathrm{H}$ & 2.086081 & -3.231411 & 0.986992 \\
$\mathrm{H}$ & -1.849392 & 3.353011 & -0.744680 \\
$\mathrm{H}$ & -1.979097 & -3.278126 & -0.744680 \\
$\mathrm{H}$ & 3.828489 & -0.074885 & -0.744680 \\
$\mathrm{H}$ & -1.059359 & 3.903402 & -2.224274 \\
$\mathrm{H}$ & -2.850766 & -2.869132 & -2.224274 \\
$\mathrm{H}$ & 3.910124 & -1.034269 & -2.224274 \\
$\mathrm{C}$ & 0.595628 & 6.214307 & 1.339754 \\
$\mathrm{C}$ & -5.679562 & -2.591324 & 1.339754 \\
$\mathrm{C}$ & 5.083934 & -3.622983 & 1.339754 \\
$\mathrm{C}$ & 0.213087 & 7.418433 & 0.753448 \\
$\mathrm{C}$ & -6.531095 & -3.524678 & 0.753448 \\
$\mathrm{C}$ & 6.318008 & -3.893755 & 0.753448 \\
$\mathrm{C}$ & -0.500799 & 7.406720 & -0.451306 \\
$\mathrm{C}$ & -6.164008 & -4.137065 & -0.451306 \\
$\mathrm{C}$ & 6.664807 & -3.269655 & -0.451306 \\
$\mathrm{C}$ & -0.816390 & 6.191038 & -1.053812 \\
$\mathrm{C}$ & -4.953401 & -3.802533 & -1.053812 \\
$\mathrm{C}$ & 5.769791 & -2.388505 & -1.053812 \\
$\mathrm{H}$ & 1.151318 & 6.224156 & 2.274154 \\
$\mathrm{H}$ & -5.965936 & -2.115007 & 2.274154 \\
$\mathrm{H}$ & 4.814618 & -4.109148 & 2.274154 \\
$\mathrm{H}$ & -1.358794 & 6.183735 & -1.995964 \\
$\mathrm{H}$ & -4.675874 & -4.268617 & -1.995964 \\
$\mathrm{H}$ & 6.034668 & -1.915117 & -1.995964 \\
$\mathrm{~S}$ & 0.529147 & 9.008081 & 1.474183 \\
$\mathrm{~S}$ & -8.5365800 & -4.045786 & 1.474183 \\
$\mathrm{C}$ & -8.000000 & -4.962295 & 1.474183 \\
$\mathrm{~S}$ & -9.98934 & 0.041987 \\
\hline
\end{tabular}




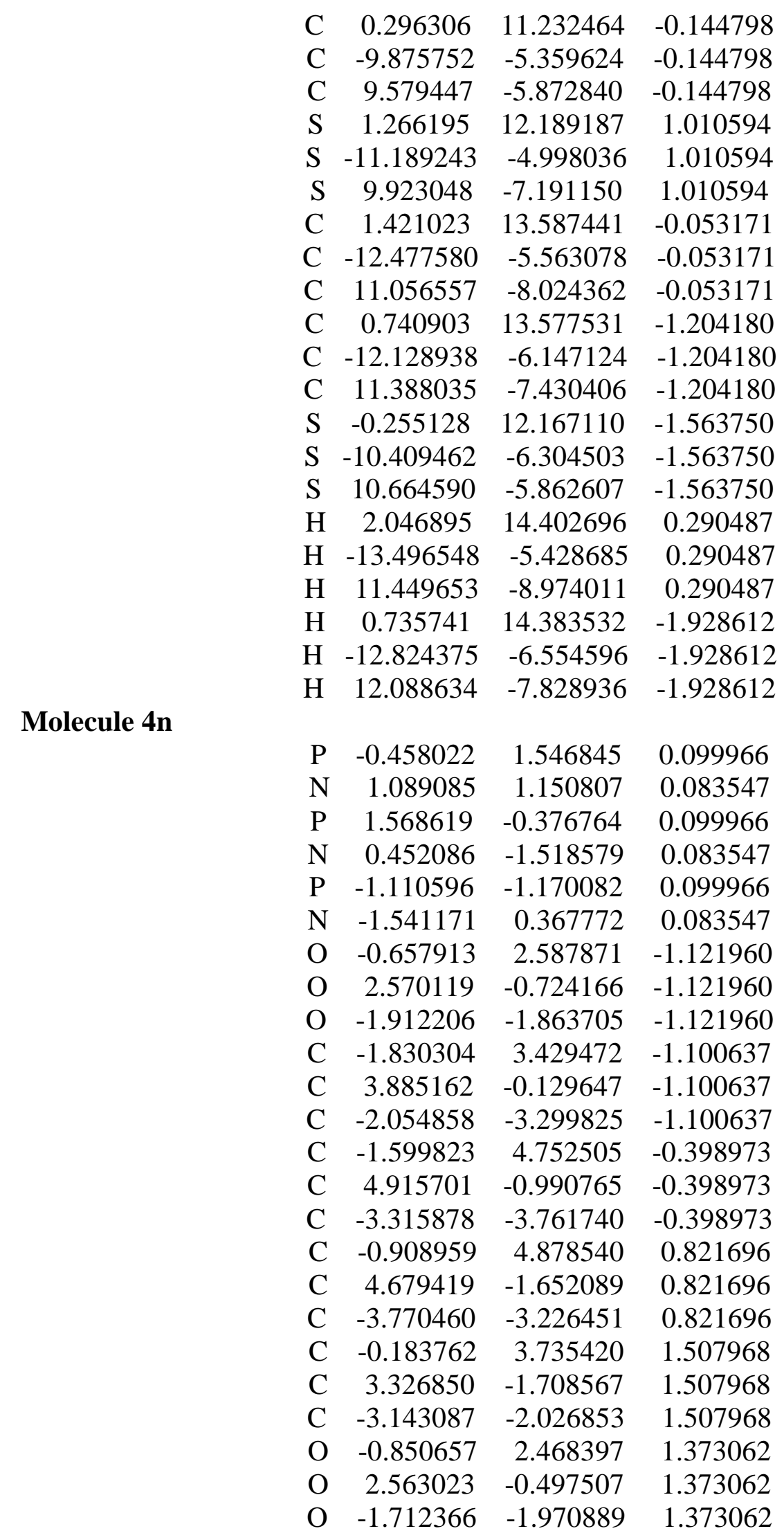




$\begin{array}{ccrc}\mathrm{H} & -2.082911 & 3.605578 & -2.148756 \\ \mathrm{H} & 4.163978 & 0.001065 & -2.148756 \\ \mathrm{H} & -2.081067 & -3.606643 & -2.148756 \\ \mathrm{H} & -2.668520 & 2.884738 & -0.649700 \\ \mathrm{H} & 3.832517 & 0.868637 & -0.649700 \\ \mathrm{H} & -1.163997 & -3.753375 & -0.649700 \\ \mathrm{H} & 0.847487 & 3.650297 & 1.141437 \\ \mathrm{H} & 2.737506 & -2.559094 & 1.141437 \\ \mathrm{H} & -3.584993 & -1.091203 & 1.141437 \\ \mathrm{H} & -0.137568 & 3.925342 & 2.583003 \\ \mathrm{H} & 3.468230 & -1.843534 & 2.583003 \\ \mathrm{H} & -3.330662 & -2.081808 & 2.583003 \\ \mathrm{C} & -2.159549 & 5.895492 & -0.981660 \\ \mathrm{C} & 6.185420 & -1.077522 & -0.981660 \\ \mathrm{C} & -4.025871 & -4.817970 & -0.981660 \\ \mathrm{C} & -2.076238 & 7.143013 & -0.367345 \\ \mathrm{C} & 7.224150 & -1.773431 & -0.367345 \\ \mathrm{C} & -5.147912 & -5.369582 & -0.367345 \\ \mathrm{C} & -1.396412 & 7.264110 & 0.850004 \\ \mathrm{C} & 6.989110 & -2.422727 & 0.850004 \\ \mathrm{C} & -5.592698 & -4.841383 & 0.850004 \\ \mathrm{C} & -0.812355 & 6.138228 & 1.422786 \\ \mathrm{C} & 5.722039 & -2.365594 & 1.422786 \\ \mathrm{C} & -4.909684 & -3.772634 & 1.422786 \\ \mathrm{H} & -2.674387 & 5.800655 & -1.934669 \\ \mathrm{H} & 6.360708 & -0.584240 & -1.934669 \\ \mathrm{H} & -3.686321 & -5.216415 & -1.934669 \\ \mathrm{He} & -0.275226 & 6.232695 & 2.363363 \\ \mathrm{H} & -9.535285 & -2.877995 & 2.363363 \\ \mathrm{H} & -5.260059 & -3.354700 & 2.363363 \\ \mathrm{Se} & -2.901621 & 8.706490 & -1.088326 \\ \mathrm{Se} & 8.990852 & -1.840368 & -1.088326 \\ \mathrm{Se} & -6.089231 & -6.866123 & -1.088326 \\ \mathrm{C} & -1.935518 & 9.858221 & 0.087602 \\ \mathrm{C} & 9.505228 & -3.252903 & 0.087602 \\ \mathrm{C} & -7.569711 & -6.605318 & 0.087602 \\ \mathrm{Se} & -1.366846 & 8.982695 & 1.685592 \\ \mathrm{Se} & 8.462665 & -3.307624 & 1.685592 \\ \mathrm{Se} & -7.095819 & -5.675071 & 1.685592 \\ \mathrm{C} & -1.720055 & 11.160189 & -0.161553 \\ \mathrm{~S} & -8.525034 & -4.090483 & -0.161553 \\ \mathrm{~S} & -8.042210 & -7.069705 & -0.161553 \\ \mathrm{H} & 13.569727 & -1.423694\end{array}$




$\begin{array}{cccr}\mathrm{C} & 12.379190 & -5.698067 & -1.423694 \\ \mathrm{C} & -11.124266 & -7.871660 & -1.423694 \\ \mathrm{C} & -0.617289 & 13.687525 & -0.256948 \\ \mathrm{C} & 12.162389 & -6.309175 & -0.256948 \\ \mathrm{C} & -11.545100 & -7.378350 & -0.256948 \\ \mathrm{Se} & -0.809850 & 12.324404 & 1.052625 \\ \mathrm{Se} & 11.078172 & -5.460852 & 1.052625 \\ \mathrm{Se} & -10.268322 & -6.863552 & 1.052625 \\ \mathrm{H} & -1.215334 & 14.312153 & -2.213179 \\ \mathrm{H} & 13.002355 & -6.103566 & -2.213179 \\ \mathrm{H} & -11.787021 & -8.208586 & -2.213179 \\ \mathrm{H} & 0.000000 & 14.537740 & 0.012602 \\ \mathrm{H} & 12.590052 & -7.268870 & 0.012602 \\ \mathrm{H} & -12.590052 & -7.268870 & 0.012602\end{array}$

\section{Molecule 4o}

$\begin{array}{lrrr}\text { C } & 0.302587 & 1.308332 & 1.888246 \\ \text { O } & 1.263599 & 0.385780 & 2.272965 \\ \text { C } & 0.981755 & -0.916214 & 1.888246 \\ \text { O } & -0.297704 & -1.287198 & 2.272965 \\ \text { C } & -1.284342 & -0.392118 & 1.888246 \\ \text { O } & -0.965894 & 0.901419 & 2.272965 \\ \text { C } & 0.582145 & 2.516975 & 1.367865 \\ \text { C } & 1.888692 & -1.762640 & 1.367865 \\ \text { C } & -2.470836 & -0.754335 & 1.367865 \\ \text { S } & -0.631191 & 3.671621 & 1.033195 \\ \text { S } & 3.495313 & -1.289183 & 1.033195 \\ \text { S } & -2.864122 & -2.382438 & 1.033195 \\ \text { C } & 0.423453 & 4.850591 & 0.477601 \\ \text { C } & 3.989008 & -2.792017 & 0.477601 \\ \text { C } & -4.412461 & -2.058574 & 0.477601 \\ \text { C } & 1.749436 & 4.543331 & 0.476345 \\ \text { C } & 3.059922 & -3.786721 & 0.476345 \\ \text { C } & -4.809358 & -0.756610 & 0.476345 \\ \text { S } & 2.179133 & 3.020791 & 1.031400 \\ \text { S } & 1.526515 & -3.397580 & 1.031400 \\ \text { S } & -3.705649 & 0.376789 & 1.031400 \\ \text { S } & 2.798672 & 5.724803 & -0.079679 \\ \text { S } & 3.558488 & -5.286123 & -0.079679 \\ \text { S } & -6.357161 & -0.438680 & -0.079679 \\ \text { C } & 1.592543 & 6.884566 & -0.416542 \\ \text { C } & 5.165938 & -4.821466 & -0.416542 \\ \text { C } & -6.758481 & -2.063100 & -0.416542 \\ \text { S } & 0.000000 & 6.374156 & -0.074876 \\ \text { S } & 5.520181 & -3.187078 & -0.074876 \\ \text { S } & -5.520181 & -3.187078 & -0.074876\end{array}$




$\begin{array}{cccc}\text { C } & 1.874196 & 8.102163 & -0.910161 \\ \mathrm{C} & 6.079581 & -5.674183 & -0.910161 \\ \mathrm{C} & -7.953777 & -2.427980 & -0.910161 \\ \mathrm{~S} & 0.684189 & 9.269973 & -1.251219 \\ \mathrm{~S} & 7.685937 & -5.227511 & -1.251219 \\ \mathrm{~S} & -8.370126 & -4.042462 & -1.251219 \\ \mathrm{C} & 1.732425 & 10.463072 & -1.805282 \\ \mathrm{C} & 8.195073 & -6.731860 & -1.805282 \\ \mathrm{C} & -9.927498 & -3.731212 & -1.805282 \\ \mathrm{C} & 3.038205 & 10.160154 & -1.807371 \\ \mathrm{C} & 7.279848 & -7.711240 & -1.807371 \\ \mathrm{C} & -10.318054 & -2.448914 & -1.807371 \\ \mathrm{~S} & 3.455756 & 8.626969 & -1.255752 \\ \mathrm{~S} & 5.743297 & -7.306257 & -1.255752 \\ \mathrm{~S} & -9.199052 & -1.320713 & -1.255752 \\ \mathrm{H} & 1.281734 & 11.405057 & -2.126493 \\ \mathrm{H} & 9.236201 & -6.812543 & -2.126493 \\ \mathrm{H} & -10.517936 & -4.592514 & -2.126493 \\ \mathrm{H} & 3.856618 & 10.807744 & -2.130569 \\ \mathrm{H} & 7.431471 & -8.743801 & -2.130569 \\ \mathrm{H} & -11.288090 & -2.063942 & -2.130569\end{array}$

Molecule 4p

$\begin{array}{rrrr}\text { C } & 0.078643 & 1.343399 & 1.547929 \\ \mathrm{O} & 1.203698 & 0.604144 & 1.831872 \\ \mathrm{C} & 1.124097 & -0.739806 & 1.547929 \\ \mathrm{O} & -0.078645 & -1.344505 & 1.831872 \\ \mathrm{C} & -1.202739 & -0.603593 & 1.547929 \\ \mathrm{O} & -1.125053 & 0.740361 & 1.831872 \\ \mathrm{C} & 0.153881 & 2.627097 & 1.179835 \\ \mathrm{C} & 2.198192 & -1.446813 & 1.179835 \\ \mathrm{C} & -2.352073 & -1.180283 & 1.179835 \\ \mathrm{~S} & -1.298628 & 3.646164 & 0.968225 \\ \mathrm{~S} & 3.806984 & -0.698437 & 0.968225 \\ \mathrm{~S} & -2.508356 & -2.947727 & 0.968225 \\ \mathrm{C} & -0.381429 & 4.960832 & 0.225646 \\ \mathrm{C} & 4.486921 & -2.150088 & 0.225646 \\ \mathrm{C} & -4.105491 & -2.810743 & 0.225646 \\ \mathrm{C} & 0.957203 & 4.882537 & 0.225249 \\ \mathrm{C} & 3.749800 & -3.270231 & 0.225249 \\ \mathrm{C} & -4.707003 & -1.612306 & 0.225249 \\ \mathrm{~S} & 1.715400 & 3.469769 & 0.967391 \\ \mathrm{~S} & 2.147208 & -3.220464 & 0.967391 \\ \mathrm{~S} & -3.862608 & -0.249304 & 0.967391 \\ \mathrm{Se} & 1.968127 & 6.294238 & -0.562568 \\ \mathrm{Se} & 4.466907 & -4.851567 & -0.562568\end{array}$




$\begin{array}{cccc}\mathrm{Se} & -6.435033 & -1.442671 & -0.562568 \\ \mathrm{C} & 0.434794 & 7.443155 & -0.482422 \\ \mathrm{C} & 6.228564 & -4.098120 & -0.482422 \\ \mathrm{C} & -6.663358 & -3.345034 & -0.482422 \\ \mathrm{Se} & -1.221418 & 6.479964 & -0.562751 \\ \mathrm{Se} & 6.222522 & -2.182203 & -0.562751 \\ \mathrm{Se} & -5.001105 & -4.297761 & -0.562751 \\ \mathrm{C} & 0.513088 & 8.781730 & -0.406703 \\ \mathrm{C} & 7.348657 & -4.835212 & -0.406703 \\ \mathrm{C} & -7.861745 & -3.946518 & -0.406703 \\ \mathrm{Se} & -1.024104 & 9.915690 & -0.352611 \\ \mathrm{Se} & 9.099292 & -4.070945 & -0.352611 \\ \mathrm{Se} & -8.075188 & -5.844745 & -0.352611 \\ \mathrm{C} & 0.000000 & 11.431164 & 0.165142 \\ \mathrm{C} & 9.899678 & -5.715582 & 0.165142 \\ \mathrm{C} & -9.899678 & -5.715582 & 0.165142 \\ \mathrm{C} & 1.332440 & 11.352996 & 0.165359 \\ \mathrm{C} & 9.165763 & -6.830425 & 0.165359 \\ \mathrm{C} & -10.498203 & -4.522571 & 0.165359 \\ \mathrm{Se} & 2.172450 & 9.727908 & -0.351555 \\ \mathrm{Se} & 7.338390 & -6.745351 & -0.351555 \\ \mathrm{Se} & -9.510840 & -2.982557 & -0.351555 \\ \mathrm{H} & -0.555282 & 12.324874 & 0.428484 \\ \mathrm{H} & 10.951295 & -5.681549 & 0.428484 \\ \mathrm{H} & -10.396013 & -6.643325 & 0.428484 \\ \mathrm{H} & 1.988384 & 12.175606 & 0.428930 \\ \mathrm{H} & 9.550192 & -7.809794 & 0.428930 \\ \mathrm{H} & -11.538576 & -4.365812 & 0.428930\end{array}$

\section{Molecule 4q}

$\begin{array}{lrrr}\mathrm{C} & 0.000000 & 0.000000 & 0.000000 \\ \mathrm{O} & 0.000000 & 0.000000 & 1.375685 \\ \mathrm{C} & 1.238018 & 0.000000 & 1.975137 \\ \mathrm{O} & 2.200425 & -0.775351 & 1.371581 \\ \mathrm{C} & 2.195319 & -0.778246 & -0.003897 \\ \mathrm{O} & 0.956293 & -0.785281 & -0.601503 \\ \mathrm{C} & -0.972140 & 0.591378 & -0.703789 \\ \mathrm{C} & 1.445059 & 0.579883 & 3.163020 \\ \mathrm{C} & 3.320641 & -0.935638 & -0.710237 \\ \mathrm{~S} & -1.075029 & 0.453769 & -2.483619 \\ \mathrm{~S} & 0.146409 & 1.446063 & 4.035156 \\ \mathrm{~S} & 4.918072 & -1.092299 & 0.077693 \\ \mathrm{C} & -2.330569 & 1.695658 & -2.587446 \\ \mathrm{C} & 1.274474 & 2.099000 & 5.231049 \\ \mathrm{C} & 5.780316 & -0.943095 & -1.459348 \\ \mathrm{C} & -2.877590 & 2.149218 & -1.448857\end{array}$




\begin{tabular}{|c|c|c|c|}
\hline & & & \\
\hline & & & \\
\hline & -2.3 & & \\
\hline & 00 & & \\
\hline & 3.309 & & \\
\hline & -4.096371 & & \\
\hline & & & \\
\hline & 5.9 & -0.7 & \\
\hline & -4.2 & & \\
\hline & 2.3 & & \\
\hline & & & \\
\hline & -2.87 & & \\
\hline & & & \\
\hline & 7.5 & -0. & \\
\hline & -5.3 & & \\
\hline & 2.5 & & \\
\hline & 8.6 & & \\
\hline & -5.4 & & \\
\hline & 1.2 & & \\
\hline & 10.2 & & \\
\hline & -7.2 & & -5 . \\
\hline & & & \\
\hline & 10.8 & & -4 \\
\hline C & -7.7 & & \\
\hline 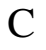 & & & 11. \\
\hline & 10. & -2 . & \\
\hline S & -6.6 & & \\
\hline ; & 4.0 & & \\
\hline S & 8.6 & -1 & \\
\hline s & -8.0 & & -7 \\
\hline 5 & & & 12. \\
\hline & 12.4 & & 204 \\
\hline 5 & -9.4 & & \\
\hline S & 4.1 & & 12. \\
\hline & 10.7 & & -7 \\
\hline  & -9.5 & & -7. \\
\hline$C$ & 2.1 & & 13. \\
\hline & 13.2 & -3 & 179 \\
\hline & -9.60 & & \\
\hline $\mathrm{C}$ & $3.5^{\prime}$ & & 13. \\
\hline & 12.5 & -2. & -7. \\
\hline & -9.5 & & -8.240537 \\
\hline & 1.776983 & 5.6 & 14.316490 \\
\hline & 14.238739 & -2.992754 & \\
\hline & -10.409339 & 3.894009 & -7.26 \\
\hline & 1.997087 & 3.888934 & 14.603 \\
\hline
\end{tabular}




$\begin{array}{cccc}\mathrm{H} & 13.333602 & -4.489810 & -6.182241 \\ \mathrm{H} & -8.823102 & 6.310904 & -6.214087 \\ \mathrm{H} & 3.744448 & 5.690633 & 12.825184 \\ \mathrm{H} & 12.518450 & -1.637268 & -6.992917 \\ \mathrm{H} & -10.578971 & 6.049763 & -6.149512 \\ \mathrm{H} & 4.195163 & 4.903266 & 14.352444 \\ \mathrm{H} & 12.983900 & -2.981810 & -8.056064\end{array}$

Molecule 4r

$\begin{array}{lrrr}\text { C } & 0.000000 & 0.000000 & 0.000000 \\ \text { O } & 0.000000 & 0.000000 & 1.375562 \\ \text { C } & 1.238550 & 0.000000 & 1.974388 \\ \text { O } & 2.197434 & -0.782492 & 1.374041 \\ \text { C } & 2.195645 & -0.781890 & -0.001676 \\ \text { O } & 0.958006 & -0.782554 & -0.602025 \\ \text { C } & -0.974269 & 0.587224 & -0.704326 \\ \text { C } & 1.448507 & 0.587276 & 3.158081 \\ \text { C } & 3.320911 & -0.942301 & -0.707474 \\ \text { S } & -1.075083 & 0.447147 & -2.484014 \\ \text { S } & 0.153497 & 1.463333 & 4.025580 \\ \text { S } & 4.917688 & -1.113711 & 0.078657 \\ \text { C } & -2.335814 & 1.683077 & -2.590654 \\ \text { C } & 1.287077 & 2.134379 & 5.205849 \\ \text { C } & 5.780888 & -0.952781 & -1.456701 \\ \text { C } & -2.887090 & 2.134593 & -1.453036 \\ \text { C } & 2.552075 & 1.685241 & 5.205131 \\ \text { C } & 5.068331 & -0.951884 & -2.594334 \\ \text { S } & -2.318399 & 1.465689 & 0.082683 \\ \text { S } & 3.007323 & 0.449985 & 4.023537 \\ \text { S } & 3.309806 & -1.111521 & -2.487646 \\ \text { S } & -4.109706 & 3.408595 & -1.573956 \\ \text { S } & 3.686863 & 2.404416 & 6.357320 \\ \text { S } & 5.941970 & -0.737353 & -4.118546 \\ \text { C } & -4.287324 & 3.185077 & -3.340877 \\ \text { C } & 2.374009 & 3.187390 & 7.288389 \\ \text { C } & 7.530264 & -1.021974 & -3.344061 \\ \text { S } & -2.878149 & 2.399477 & -4.115574 \\ \text { S } & 0.860456 & 3.407560 & 6.358992 \\ \text { S } & 7.533861 & -0.739684 & -1.576747 \\ \text { C } & -5.369934 & 3.604368 & -4.031530 \\ \text { C } & 2.524024 & 3.606902 & 8.563674 \\ \text { C } & 8.633629 & -1.381004 & -4.035820 \\ \text { S } & -5.502250 & 3.421416 & -5.803437 \\ \text { S } & 1.218002 & 4.436629 & 9.456141 \\ \text { S } & 10.222254 & -1.605553 & -3.250654 \\ \text { C } & -7.232831 & 3.810456 & -5.812165\end{array}$




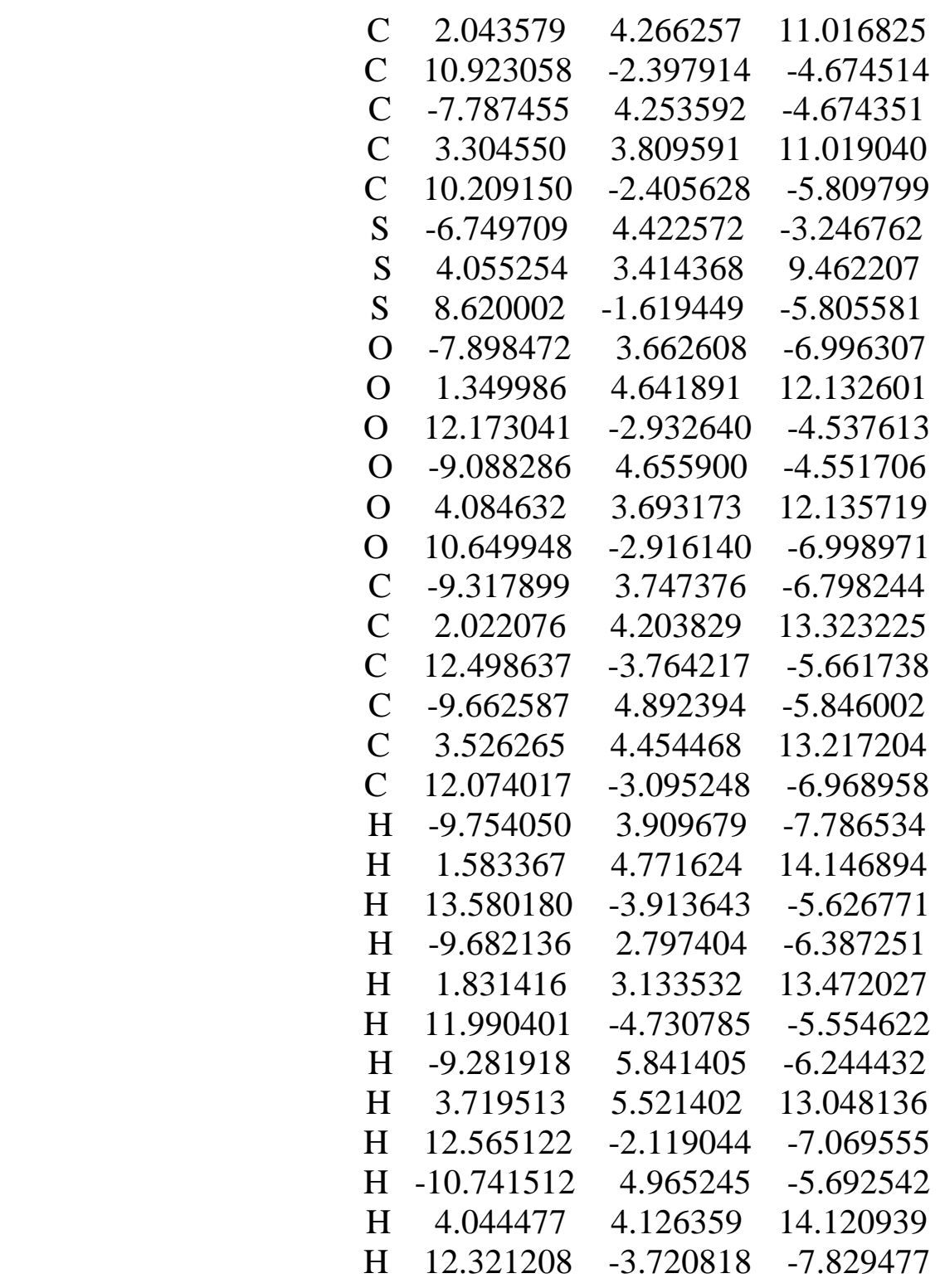

\section{TPP}

$\begin{array}{lrrr}\mathrm{P} & 1.618529 & 0.000000 & 0.000000 \\ \mathrm{~N} & 0.787217 & -1.363499 & -0.002413 \\ \mathrm{P} & -0.809265 & -1.401687 & 0.000000 \\ \mathrm{~N} & -1.574433 & 0.000000 & -0.002413 \\ \mathrm{P} & -0.809265 & 1.401687 & 0.000000 \\ \mathrm{~N} & 0.787217 & 1.363499 & -0.002413 \\ \mathrm{O} & 2.724776 & 0.000000 & -1.217412 \\ \mathrm{O} & -1.362388 & -2.359725 & -1.217412 \\ \mathrm{O} & -1.362388 & 2.359725 & -1.217412 \\ \mathrm{C} & 4.008527 & 0.000000 & -0.690064 \\ \mathrm{C} & -2.004263 & -3.471486 & -0.690064 \\ \mathrm{C} & -2.004263 & 3.471486 & -0.690064\end{array}$




$\begin{array}{rrrr}\mathrm{C} & 4.004593 & 0.000000 & 0.703943 \\ \mathrm{C} & -2.002297 & -3.468080 & 0.703943 \\ \mathrm{C} & -2.002297 & 3.468080 & 0.703943 \\ \mathrm{O} & 2.718101 & 0.000000 & 1.224137 \\ \mathrm{O} & -1.359050 & -2.353944 & 1.224137 \\ \mathrm{O} & -1.359050 & 2.353944 & 1.224137 \\ \mathrm{C} & 5.185319 & 0.000000 & -1.414710 \\ \mathrm{C} & -2.592659 & -4.490618 & -1.414710 \\ \mathrm{C} & -2.592659 & 4.490618 & -1.414710 \\ \mathrm{C} & 6.383482 & 0.000000 & -0.684952 \\ \mathrm{C} & -3.191741 & -5.528257 & -0.684952 \\ \mathrm{C} & -3.191741 & 5.528257 & -0.684952 \\ \mathrm{C} & 6.379600 & 0.000000 & 0.711756 \\ \mathrm{C} & -3.189800 & -5.524896 & 0.711756 \\ \mathrm{C} & -3.189800 & 5.524896 & 0.711756 \\ \mathrm{C} & 5.177478 & 0.000000 & 1.434957 \\ \mathrm{C} & -2.588739 & -4.483828 & 1.434957 \\ \mathrm{C} & -2.588739 & 4.483828 & 1.434957 \\ \mathrm{H} & 5.170128 & 0.000000 & -2.499056 \\ \mathrm{H} & -2.585064 & -4.477462 & -2.499056 \\ \mathrm{H} & -2.585064 & 4.477462 & -2.499056 \\ \mathrm{H} & 7.328033 & 0.000000 & -1.220353 \\ \mathrm{H} & -3.664016 & -6.346263 & -1.220353 \\ \mathrm{H} & -3.664016 & 6.346263 & -1.220353 \\ \mathrm{H} & 7.321193 & 0.000000 & 1.252355 \\ \mathrm{H} & -3.660596 & -6.340339 & 1.252355 \\ \mathrm{H} & -3.660596 & 6.340339 & 1.252355 \\ \mathrm{H} & 5.156433 & 0.000000 & 2.519205 \\ \mathrm{H} & -2.578217 & -4.465602 & 2.519205 \\ \mathrm{H} & -2.578217 & 4.465602 & 2.519205\end{array}$


II. Figures not given in the text.

$1 f$

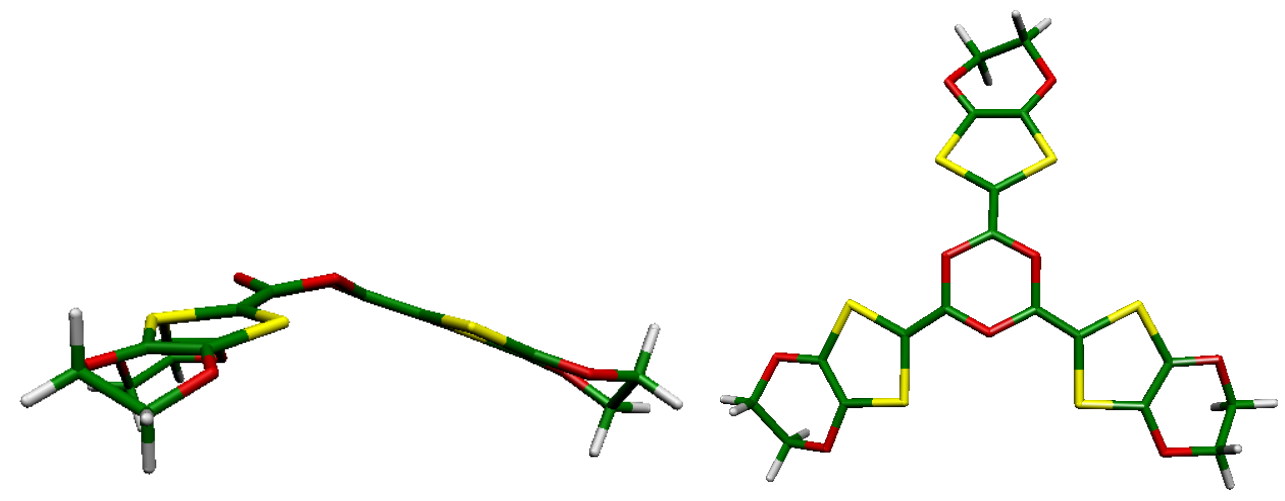

$2 f$



3f
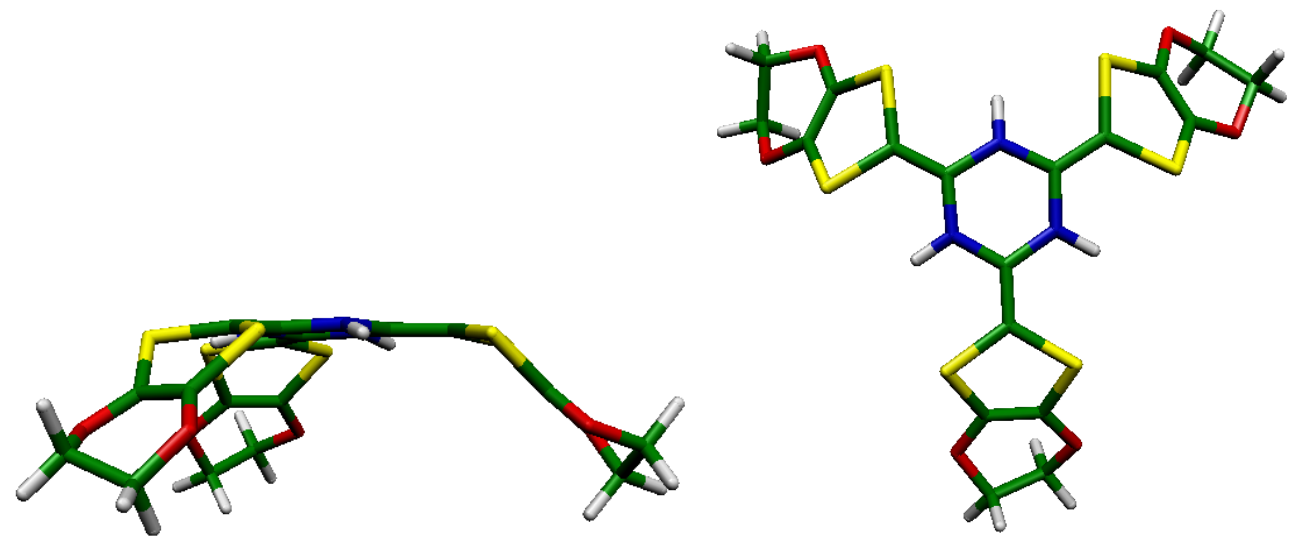

4f 


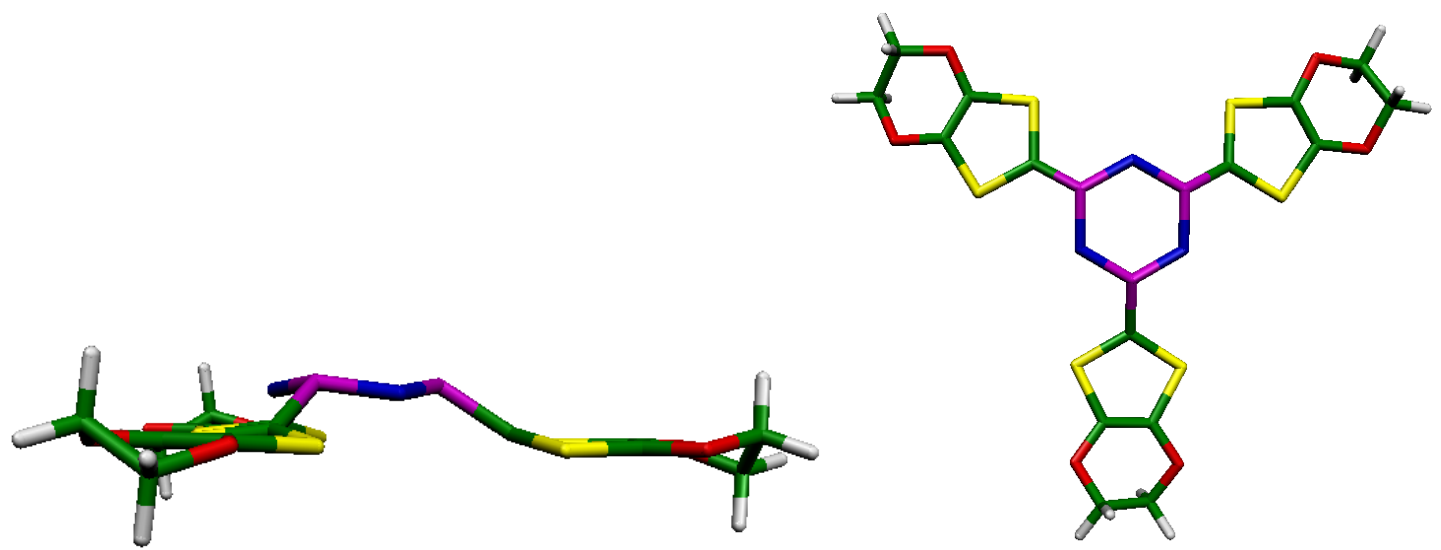

Figure S1. Selected B3LYP/6-31G* 3D optimized geometries of representative models. The plots in the first and second columns correspond to the side (left column) and top (right column) view of the molecules (H, C, N, O, P, S and Se are shown in White, Green, Blue, Red, Light Purple and Orange colors respectively).

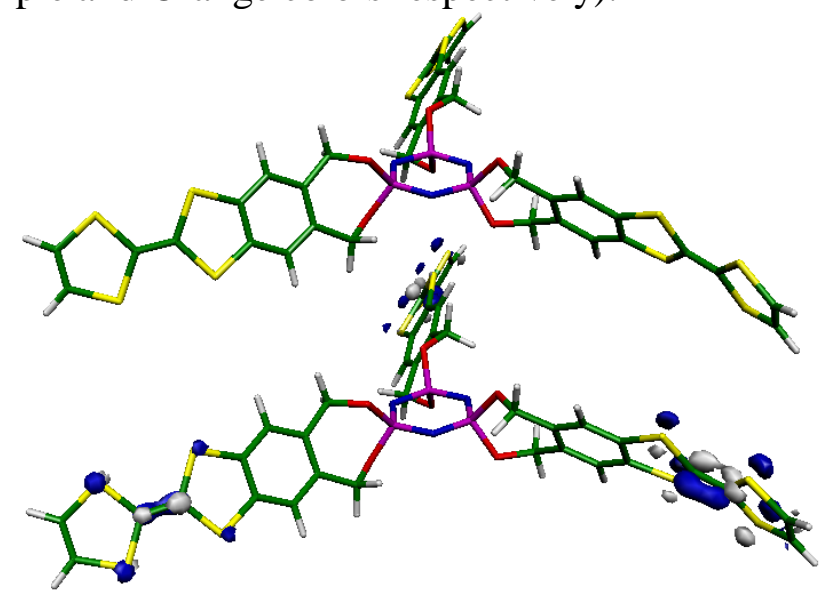

Figure S2. (a) The B3LYP/6-31G* optimized structure of HOT2 and (b) the corresponding highest occupied molecular orbital (contoured at $0.05 \mathrm{au}$ )

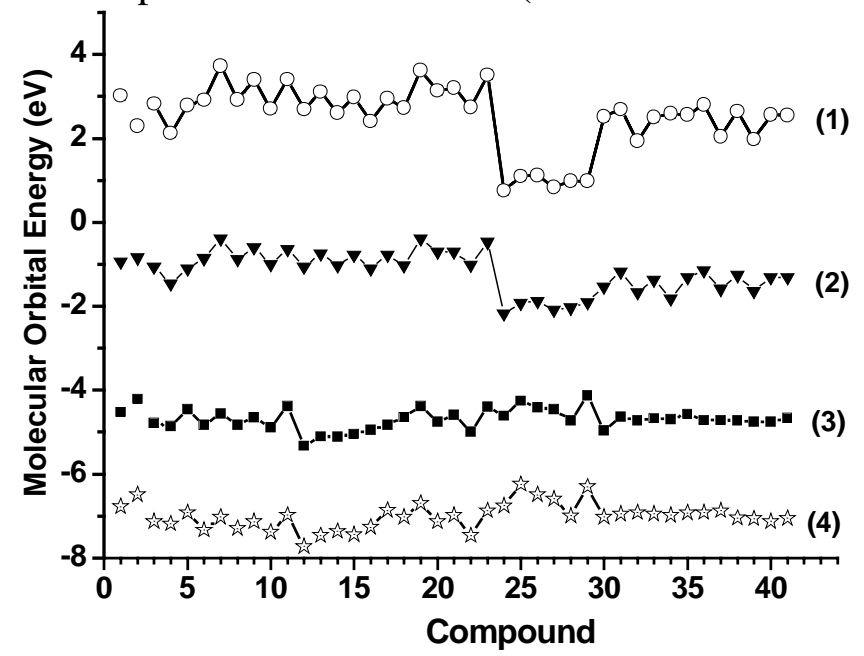

Figure S3. Frontier molecular orbital energy evolution among the series of system considered: (1) HF/6-31G** $\mathrm{E}_{\mathrm{LUMO}}$, (2) for B3LYP/6-31G* $\mathrm{E}_{\mathrm{LUMO}}$, (3) for B3LYP/6$31 \mathrm{G}^{*} \mathrm{E}_{\mathrm{HOMO}}$ and (4) for $\mathrm{HF} / 6-31 \mathrm{G}^{* *} \mathrm{E}_{\mathrm{HO} \mathrm{O}}$. 
III. Tables: computational (numerical) results not given in the text.

Table S1: B3LYP/6-31G* based energetics and spin contamination $\left(\mathrm{S}^{* * 2}\right.$ in both relaxed and non relaxed cationic forms)

\begin{tabular}{|c|c|c|c|c|c|}
\hline System & $\mathrm{E}_{\mathrm{M}+(\text { nopt })}$ & $\mathrm{E}_{\mathrm{M}+(\mathrm{opt})}$ & $\mathrm{E}_{\mathrm{M}(\mathrm{opt})}$ & $\left\langle\mathrm{S}^{* * 2}\right\rangle_{\mathrm{M}+(\text { nopt })}$ & $\left\langle\mathrm{S}^{* * 2}\right\rangle_{\mathrm{M}+(\mathrm{opt})}$ \\
\hline TTF (p) & -1823.504939 & -1823.50994985 & -1823.73591277 & 0.753035 & 0.752734 \\
\hline TTF (d) & -1823.504842 & -1823.509908 & -1823.735913 & 0.753151 & 0.752738 \\
\hline TMTSF (p) & -9985.5573489 & -9985.5626973 & -9985.77137822 & 0.752450 & 0.752150 \\
\hline TMTSF (d) & -9985.552254 & -9985.562614 & -9985.776291 & 0.752927 & 0.752155 \\
\hline BEDT-TTF & -3571.098065 & -3571.10821 & -3571.325922 & 0.753298 & 0.752489 \\
\hline BETS-TTF & -11575.86769 & -11575.87602 & -11576.09705 & 0.753302 & 0.752503 \\
\hline BEDO-TTF & -2279.173672 & -2279.184479 & -2279.395382 & 0.753227 & 0.752838 \\
\hline $1 \mathbf{a}$ & -5700.3033469 & -5700.3139978 & -5700.5222904 & 0.752366 & 0.755131 \\
\hline $1 b$ & -3075.3057639 & -3075.3157651 & -3075.5226285 & 0.752333 & 0.755646 \\
\hline 1c & -15082.4386132 & -15082.4539155 & -15082.6639222 & 0.752149 & 0.754618 \\
\hline 1d & -5696.693197 & -5696.7021532 & -5696.9050825 & 0.752394 & 0.754517 \\
\hline 1e & -17703.8430678 & -17703.8578811 & -17704.0641746 & 0.751854 & 0.753644 \\
\hline 1f & -3758.8062959 & -3758.8147873 & -3759.0115416 & 0.752104 & 0.754398 \\
\hline $2 \mathbf{a}$ & -6669.2224328 & -6669.2368265 & -6669.4589263 & 0.753175 & 0.753005 \\
\hline $2 b$ & -4044.225923 & -4044.235375 & -4044.46752334 & 0.753655 & 0.752721 \\
\hline 2c & -16051.3691415 & -16051.3723342 & -16051.6063777 & 0.757538 & 0.757065 \\
\hline 2d & -6665.616065 & -6665.8410512 & -6663.6245163 & 0.753303 & 0.752629 \\
\hline $2 e$ & -18672.7800705 & -18672.7879109 & -18673.0056732 & 0.753909 & 0.754634 \\
\hline $2 f$ & -4727.7371068 & -4727.9501315 & -4727.7370785 & 0.753192 & 0.753193 \\
\hline $\mathbf{3 a}$ & -5640.7485782 & -5640.7604883 & -5640.9599751 & 0.762934 & 0.764875 \\
\hline $3 b$ & -3015.7426624 & -3015.7587395 & -3015.9515917 & 0.764339 & 0.763941 \\
\hline $3 c$ & -15022.8743716 & -15022.8912476 & -15023.095827 & 0.765061 & 0.763007 \\
\hline 3d & -5637.1380611 & -5637.1475356 & -5637.34720588 & 0.760816 & 0.763272 \\
\hline $3 e$ & -17644.29244 & -17644.3055157 & -17644.5149275 & 0.756563 & 0.760983 \\
\hline 3f & -3699.2411788 & -3699.2536515 & -3699.44654550 & 0.760218 & 0.762232 \\
\hline $4 \mathbf{a}$ & -6548.616284 & -6548.6306465 & -6548.82433619 & 0.774805 & 0.765223 \\
\hline $4 b$ & -3923.6171056 & -3923.6270633 & -3923.81882299 & 0.777906 & 0.772139 \\
\hline $4 c$ & -15930.7561194 & -15930.7677597 & -15930.9620801 & 0.770912 & 0.776872 \\
\hline 4d & -6545.0046547 & -6545.015990 & -6545.2073838 & 0.760500 & 0.767431 \\
\hline $4 e$ & -18552.1623886 & -18552.1738719 & -18552.3727268 & 0.768598 & 0.750431 \\
\hline $4 f$ & -4607.116792 & -4607.1283644 & -4607.31040934 & 0.772877 & 0.762565 \\
\hline $4 g$ & -10193.1529144 & -10193.1546116 & -10193.35579 & 0.751223 & 0.751261 \\
\hline $4 h$ & -7568.1538373 & -7568.1556314 & -7568.3563552 & 0.751220 & 0.751222 \\
\hline $4 \mathbf{i}$ & -31582.4427186 & -31582.4438932 & -31582.6441841 & 0.751049 & 0.751042 \\
\hline $\mathbf{4 j}$ & -10189.5370664 & -10189.5385748 & -10189.735265 & 0.751087 & 0.751362 \\
\hline $4 k$ & -34204.8424841 & -34204.843594 & -34204.040610 & 0.751251 & 0.751142 \\
\hline 41 & -8251.8691302 & -8251.6511505 & -8251.8445512 & 0.751242 & 0.751209 \\
\hline $4 m$ & -7804.0332939 & -7804.0381795 & -7804.2387952 & 0.751275 & 0.751180 \\
\hline $4 n$ & -31818.2780754 & na & -31818.5264779 & 0.750995 & na \\
\hline 40 & -8310.7074132 & -8310.7106984 & -8310.9134062 & 0.751072 & 0.751166 \\
\hline $4 p$ & -32325.0066496 & -32325.010737 & -32325.2102593 & 0.751205 & 0.752347 \\
\hline $4 q$ & -10932.092301 & -10932.0953254 & -10932.2968323 & 0.751052 & 0.751197 \\
\hline
\end{tabular}




\begin{tabular}{cccccc}
\hline $\mathbf{4 r}$ & -8994.2009282 & -8994.204417 & -8994.402273 & 0.751070 & 0.751012 \\
$\mathbf{T P P}$ & -2332.6612467 & -2332.6635625 & -2332.9375629 & 0.753865 & 0.753892 \\
\hline
\end{tabular}

$\mathrm{E}_{\mathrm{M}+(\mathrm{nopt})}=$ cation energy in the optimized geometry of the neutral form; $\mathrm{E}_{\mathrm{M}+(\mathrm{opt})}=$ cation energy in its optimized geometry; $\mathrm{E}_{\mathrm{M}(\mathrm{opt})}=$ molecular energy in the optimized geometry of the neutral form. 
Table S2. Structural parameters of BEDT, BEDT ${ }^{+}$and BEDT ${ }^{+1 / 2}$ from the B3LYP/6$31 \mathrm{G}^{*}$ calculations along with available theoretical and experimental data (see chart $1 \mathrm{ii}$ for the definition of the parameters).

\begin{tabular}{|c|c|c|c|c|c|c|c|c|c|}
\hline & BEDT & BEDT $^{+}$ & $\mathrm{BEDT}^{+1 / 2 a}$ & Calc. $^{b}$ & Calc. ${ }^{c}$ & Calc. $^{d}$ & Exp. $^{e}$ & Exp..$^{f}$ & Exp. $^{g}$ \\
\hline \multicolumn{10}{|c|}{ Bond distances $(\AA)$} \\
\hline $\mathrm{C}-\mathrm{C}_{1}$ & 1.350 & 1.386 & 1.368 & 1.326 & 1.389 & 1.358 & 1.381 & 1.383 & 1.360 \\
\hline $\mathrm{C}_{1}-\mathrm{S}_{1}$ & 1.781 & 1.747 & 1.764 & 1.773 & 1.721 & 1.747 & 1.721 & 1.733 & 1.741 \\
\hline $\mathrm{S}_{1}-\mathrm{C}_{2}$ & 1.784 & 1.762 & 1.773 & 1.771 & 1.751 & 1.761 & 1.732 & 1.742 & 1.751 \\
\hline $\mathrm{C}_{2}-\mathrm{C}$ & 1.349 & 1.365 & 1.357 & 1.322 & 1.336 & 1.329 & 1.351 & 1.371 & 1.343 \\
\hline $\mathrm{C}_{2}-\mathrm{S}_{2}$ & 1.763 & 1.763 & 1.763 & 1.769 & 1.765 & 1.767 & 1.736 & 1.741 & 1.749 \\
\hline $\mathrm{S}_{2}-\mathrm{C}_{3}$ & 1.843 & 1.837 & 1.840 & 1.812 & 1.816 & 1.814 & 1.809 & 1.818 & 1.811 \\
\hline $\mathrm{C}_{3}-\mathrm{C}$ & 1.521 & 1.525 & 1.523 & 1.524 & 1.523 & 1.524 & 1.381 & 1.497 & 1.485 \\
\hline \multicolumn{10}{|c|}{ Bond Angles (deg) } \\
\hline $\mathrm{C}-\mathrm{C}_{1}-\mathrm{S}_{1}$ & 123.3 & 122.8 & 123.05 & 123.22 & 122.76 & 122.99 & 122.5 & & 122.4 \\
\hline $\mathrm{C}_{1}-\mathrm{S}_{1}-\mathrm{C}_{2}$ & 93.7 & 96.3 & 95.0 & 95.57 & 96.33 & 95.95 & 95.9 & & 95.1 \\
\hline $\mathrm{S}_{1}-\mathrm{C}_{2}-\mathrm{C}$ & 116.8 & 116.4 & 116.6 & 117.65 & 116.42 & 117.04 & 116.6 & & 117.1 \\
\hline $\mathrm{C}-\mathrm{C}_{2}-\mathrm{S}_{2}$ & 127.7 & 128.8 & 128.25 & 128.68 & 128.83 & 128.76 & 126.9 & & 128.9 \\
\hline $\mathrm{C}_{2}-\mathrm{S}_{2}-\mathrm{C}_{3}$ & 103.8 & 100.6 & 102.2 & 100.86 & 100.50 & 100.68 & 100.6 & & 100.9 \\
\hline $\mathrm{S}_{2}-\mathrm{C}_{3}-\mathrm{C}$ & 115.6 & 113.3 & 114.45 & 112.93 & 112.68 & 112.80 & 115.0 & & 115.1 \\
\hline
\end{tabular}

Table S3. Computational results for TTF, BEDT and BEDO along with data from previous theoretical studies and experiments for comparison.

\begin{tabular}{ccccc}
\hline System & $\mathrm{IPv}^{a}$ & $\mathrm{IPa}^{b}$ & $\mathrm{IP}_{\mathrm{KT}}{ }^{c}$ & Exp. \\
\hline TTF & 6.29 & $6.15,5.82^{d}$ & $6.77,6.80^{d}$ & $6.7^{e} ; 6.4^{f}$ \\
BEDT & $6.2,6.11^{g}$, & $5.92,5.77^{d}$, & $7.12,6.87^{d}$, & $6.7^{e} ; 6.21^{f}$ \\
& $6.15^{g}$ & $5.82^{g}, 5.83^{g}$ & $6.82^{g}, 6.83^{g}$ & \\
BEDO & 6.03 & $5.74,5.725^{d}$ & $6.91,6.791^{d}$ & $6.46^{e}$ \\
\hline
\end{tabular}

${ }^{a}$ Vertical Ionization Potential $(\mathrm{eV}) ;{ }^{b}$ Adiabatic Ionization Potential $(\mathrm{eV}) ;{ }^{c}$ Ionization Potential from Koopmans theorem (eV); ${ }^{d}$ Data from reference 10(a) (for BEDT) and 10(d); ${ }^{e}$ Data from reference $43 ;{ }^{f}$ Data from reference $44 ;{ }^{g}$ Data from reference 39 ; In all cases, a better agreement with experimental values is provided by the current results; Exp. $=$ experimental. 
Table S4: B3LYP five-membered ring $\theta(=\mathrm{C}-\mathrm{X}-\mathrm{C}$ with $\mathrm{X}=\mathrm{S}$ or Se) bond angle in the neutral form.

\begin{tabular}{|c|c|c|c|c|c|}
\hline System & $\theta 1$ & $\theta 2$ & $\theta 3$ & $\theta 4$ & $\theta_{\mathrm{av}}$ \\
\hline TTF (d) & 94.743 & 94.745 & & & 94.744 \\
\hline TMTSF (d) & 93.406 & 93.407 & & & 93.407 \\
\hline BEDT & 93.651 & 93.728 & & & 93.690 \\
\hline BETS & 91.361 & 91.411 & & & 91.386 \\
\hline BEDO & 92.965 & $\begin{array}{c}92.972 \\
{\left[(\mathbf{C O})_{3}\right]}\end{array}$ & & & 92.969 \\
\hline $1 \mathrm{a}$ & 94.559 & 94.552 & & & 94.556 \\
\hline $1 b$ & 94.082 & 94.087 & & & 94.085 \\
\hline $1 c$ & 92.023 & 92.026 & & & 92.025 \\
\hline $1 d$ & 93.224 & 93.326 & & & 93.275 \\
\hline $1 e$ & 91.070 & 91.125 & & & 91.098 \\
\hline 1f & 92.685 & $\begin{array}{c}92.692 \\
{\left[(\mathbf{C S})_{3}\right]}\end{array}$ & & & 92.689 \\
\hline $2 \mathbf{a}$ & 96.023 & 96.047 & & & 96.035 \\
\hline $2 b$ & 95.321 & 95.322 & & & 95.322 \\
\hline $2 c$ & 93.206 & 93.210 & & & 93.208 \\
\hline 2d & 95.110 & 95.196 & & & 95.153 \\
\hline $2 e$ & 93.135 & 93.199 & & & 93.167 \\
\hline $2 f$ & 94.072 & $\begin{array}{l}94.071 \\
{[(\mathbf{C N H})}\end{array}$ & & & 94.072 \\
\hline $3 a$ & 92.667 & 92.976 & & & 92.822 \\
\hline $3 b$ & 92.689 & 92.690 & & & 92.690 \\
\hline $3 c$ & 89.670 & 89.679 & & & 89.675 \\
\hline $3 d$ & 91.800 & 91.765 & & & 91.783 \\
\hline $3 e$ & 88.486 & 88.542 & & & 88.514 \\
\hline $3 f$ & 90.803 & $\begin{array}{c}90.737 \\
{\left[(\mathbf{N P})_{3}\right]}\end{array}$ & & & 90.770 \\
\hline $4 a$ & 95.284 & 95.277 & & & 95.281 \\
\hline $4 b$ & 95.122 & 95.124 & & & 95.123 \\
\hline $4 c$ & 92.475 & 92.467 & & & 92.471 \\
\hline $4 d$ & 94.557 & 94.619 & & & 94.588 \\
\hline $4 e$ & 91.277 & 91.228 & & & 91.253 \\
\hline $4 f$ & 93.909 & 93.897 & & & 93.903 \\
\hline $4 g$ & 95.824 & 95.822 & 96.051 & 96.052 & 95.937 \\
\hline $4 h$ & 94.675 & 94.675 & 94.676 & 94.676 & 94.676 \\
\hline $4 \mathbf{i}$ & 92.132 & 92.134 & 92.702 & 92.701 & 92.417 \\
\hline
\end{tabular}




\begin{tabular}{cccccc}
\hline $\mathbf{4 j}$ & 95.817 & 95.816 & 95.445 & 95.445 & 95.631 \\
$\mathbf{4 k}$ & 92.899 & 92.918 & 90.967 & 90.922 & 91.927 \\
$\mathbf{4 l}$ & 95.814 & 95.810 & 93.712 & 93.709 & 94.761 \\
$\mathbf{4 m}$ & 95.699 & 95.698 & 94.994 & 94.991 & 95.346 \\
$\mathbf{4 n}$ & 94.270 & 94.269 & 93.341 & 93.341 & 93.805 \\
$\mathbf{4 o}$ & 92.625 & 92.626 & 94.664 & 94.662 & 93.644 \\
$\mathbf{4 p}$ & 90.136 & 90.136 & 92.718 & 92.718 & 91.427 \\
$\mathbf{4 q}$ & 92.881 & 92.873 & 93.738 & 93.659 & 93.288 \\
$\mathbf{4 r}$ & 93.533 & 93.535 & 93.922 & 93.932 & 93.731 \\
\hline
\end{tabular}

$* \theta_{\mathrm{av} .}$ is the average value: (d) refers to the distorted conformation.

Table S5: DFT electronic properties calculation results for a series of TTF and TPP modified models: frontier molecular energy, LUMO-HOMO $(\triangle \mathrm{E})$ and HOMO-SOMO $\left(\Delta \mathrm{E}^{\prime}\right)$ energy gap.*

\begin{tabular}{|c|c|c|c|c|c|}
\hline System & LUMO & HOMO & SOMO & $\Delta \mathrm{E}$ & $\Delta \mathrm{E}^{\prime}$ \\
\hline TTF (p) & -0.03471 & -0.16599 & -0.34177 & 0.13128 & 0.17578 \\
\hline TTF (d) & -0.03472 & -0.16600 & -0.34178 & 0.13128 & 0.17578 \\
\hline TMTSF (p) & -0.03098 & -0.15459 & -0.31731 & 0.12361 & 0.16272 \\
\hline TMTSF (d) & -0.04320 & -0.16670 & -0.31580 & 0.12350 & 0.14910 \\
\hline BEDT & -0.03898 & -0.17567 & -0.30852 & 0.13669 & 0.13285 \\
\hline BETS & -0.05350 & -0.17868 & -0.30757 & 0.12518 & 0.12889 \\
\hline BEDO & -0.04030 & -0.16375 & -0.31022 & 0.12345 & 0.14647 \\
\hline $1 \mathbf{a}$ & -0.03174 & -0.17754 & -0.27905 & 0.14580 & 0.10151 \\
\hline $1 b$ & -0.01402 & -0.16769 & -0.28729 & 0.15367 & 0.11960 \\
\hline 1c & -0.03250 & -0.17696 & -0.28331 & 0.14446 & 0.10635 \\
\hline 1d & -0.02208 & -0.17097 & -0.26881 & 0.14889 & 0.09784 \\
\hline 1e & -0.03688 & -0.17097 & -0.27022 & 0.13409 & 0.09925 \\
\hline 1f & -0.02355 & -0.16085 & -0.26740 & 0.13730 & 0.10655 \\
\hline $2 \mathbf{a}$ & -0.03889 & -0.19570 & -0.28559 & 0.15681 & 0.08989 \\
\hline $2 b$ & -0.02718 & -0.18732 & -0.30072 & 0.16014 & 0.11340 \\
\hline 2c & -0.03789 & -0.18790 & -0.31047 & 0.15001 & 0.12257 \\
\hline 2d & -0.02846 & -0.18539 & -0.28604 & 0.15693 & 0.10065 \\
\hline $2 e$ & -0.04048 & -0.18195 & -0.28719 & 0.14147 & 0.10524 \\
\hline $2 f$ & -0.02829 & -0.17766 & -0.28113 & 0.14937 & 0.10347 \\
\hline $\mathbf{3 a}$ & -0.03784 & -0.17065 & -0.27018 & 0.13281 & 0.09953 \\
\hline $3 b$ & -0.01427 & -0.16092 & -0.27428 & 0.14665 & 0.11336 \\
\hline $3 c$ & -0.02574 & -0.17493 & -0.27751 & 0.14919 & 0.10258 \\
\hline 3d & -0.02581 & -0.16883 & -0.26854 & 0.14302 & 0.09971 \\
\hline $3 e$ & -0.03722 & -0.18329 & -0.27265 & 0.14607 & 0.08936 \\
\hline $3 f$ & -0.01719 & -0.16135 & -0.26245 & 0.14416 & 0.10110 \\
\hline $4 a$ & -0.08027 & -0.16953 & -0.26065 & 0.08926 & 0.09112 \\
\hline $4 b$ & -0.07072 & -0.15612 & -0.27265 & 0.08540 & 0.11653 \\
\hline $4 c$ & -0.06886 & -0.16192 & -0.28631 & 0.09306 & 0.12439 \\
\hline 4d & -0.07678 & -0.16376 & -0.25944 & 0.08698 & 0.09568 \\
\hline
\end{tabular}




\begin{tabular}{cccccc}
\hline $\mathbf{4 e}$ & -0.07468 & -0.17341 & -0.26066 & 0.09873 & 0.08725 \\
$\mathbf{4 f}$ & -0.06976 & -0.15185 & -0.25494 & 0.08209 & 0.10309 \\
$\mathbf{4 g}$ & -0.05634 & -0.18243 & -0.25417 & 0.12609 & 0.07174 \\
$\mathbf{4 h}$ & -0.04284 & -0.17008 & -0.25038 & 0.12724 & 0.08030 \\
$\mathbf{4 i}$ & -0.06119 & -0.17328 & -0.24778 & 0.11209 & 0.07450 \\
$\mathbf{4 j}$ & -0.05028 & -0.17179 & -0.24481 & 0.12151 & 0.07302 \\
$\mathbf{4 k}$ & -0.06689 & -0.17240 & -0.24306 & 0.10551 & 0.07066 \\
$\mathbf{4 l}$ & -0.04865 & -0.16779 & -0.24390 & 0.11914 & 0.07611 \\
$\mathbf{4 m}$ & -0.03965 & -0.17750 & -0.24767 & 0.13785 & 0.07017 \\
$\mathbf{4 n}$ & -0.05359 & -0.18079 & na & 0.1272 & na \\
$\mathbf{4 o}$ & -0.04618 & -0.17344 & -0.25322 & 0.12726 & 0.07978 \\
$\mathbf{4 p}$ & -0.06045 & -0.17459 & -0.25467 & 0.11414 & 0.08008 \\
$\mathbf{4 q}$ & -0.04825 & -0.17510 & -0.24865 & 0.12685 & 0.07355 \\
$\mathbf{4 r}$ & -0.04850 & -0.17125 & -0.24415 & 0.12275 & 0.07290 \\
$\mathbf{T P P}$ & -0.01063 & -0.23248 & -0.35611 & 0.22185 & 0.12363 \\
\hline
\end{tabular}

* Energies are given in a.u $(1 \mathrm{a} . \mathrm{u}=27.2114 \mathrm{eV})$; na: calculation not achieved 
Table S6: HF/6-31G**//B3LYP/6-31G* electronic properties calculation results for a series of TTF and TPP modified models: frontier molecular energy, LUMO-HOMO $(\triangle \mathrm{E})$ and $\mathrm{IP}_{\mathrm{KT}}$.

\begin{tabular}{|c|c|c|c|c|}
\hline System & HOMO & LUMO & $\Delta \mathrm{E}$ & $\mathrm{IP}_{\mathrm{KT}}$ \\
\hline TTF (p) & -0.24888 & 0.11109 & 0.35997 & 0.24888 \\
\hline TTF (d) & -0.24890 & 0.11111 & 0.36001 & 0.24890 \\
\hline TMTSF (p) & -0.23837 & 0.08433 & 0.32270 & 0.23837 \\
\hline TMTSF (d) & -0.24880 & 0.08962 & 0.33842 & 0.24880 \\
\hline BEDT & -0.26155 & 0.10336 & 0.36491 & 0.26155 \\
\hline BETS & -0.26349 & 0.07791 & 0.34140 & 0.26349 \\
\hline BEDO & -0.25406 & 0.10265 & 0.35671 & 0.25406 \\
\hline 1a & -0.26955 & 0.10697 & 0.37652 & 0.26955 \\
\hline 1b & -0.25807 & 0.13701 & 0.39508 & 0.25807 \\
\hline 1c & -0.2679 & 0.10731 & 0.37521 & 0.26790 \\
\hline 1d & -0.26215 & 0.12499 & 0.38714 & 0.26215 \\
\hline 1e & -0.27112 & 0.09958 & 0.37070 & 0.27112 \\
\hline 1f & -0.25606 & 0.12527 & 0.38133 & 0.25606 \\
\hline $2 \mathbf{a}$ & -0.28359 & 0.09888 & 0.38247 & 0.28359 \\
\hline $2 \mathbf{b}$ & -0.27416 & 0.11426 & 0.38842 & 0.27416 \\
\hline $2 c$ & -0.27055 & 0.09607 & 0.36662 & 0.27055 \\
\hline $2 d$ & -0.27336 & 0.10964 & 0.38300 & 0.27336 \\
\hline $2 e$ & -0.26567 & 0.09023 & 0.35590 & 0.26567 \\
\hline 2f & -0.25199 & 0.10825 & 0.36024 & 0.25199 \\
\hline $3 \mathbf{a}$ & -0.25786 & 0.10039 & 0.35825 & 0.25786 \\
\hline 3b & -0.24583 & 0.13300 & 0.37883 & 0.24583 \\
\hline $3 c$ & -0.26199 & 0.11523 & 0.37722 & 0.26199 \\
\hline 3d & -0.25663 & 0.11750 & 0.37413 & 0.25663 \\
\hline $3 e$ & -0.27410 & 0.10084 & 0.37494 & 0.27410 \\
\hline 3f & -0.25247 & 0.12896 & 0.38143 & 0.25247 \\
\hline $4 \mathbf{a}$ & -0.24797 & 0.02779 & 0.27576 & 0.24797 \\
\hline $4 b$ & -0.22943 & 0.04060 & 0.27003 & 0.22943 \\
\hline $4 c$ & -0.23833 & 0.04114 & 0.27947 & 0.23833 \\
\hline $4 d$ & -0.24204 & 0.03090 & 0.27294 & 0.24204 \\
\hline $4 e$ & -0.25685 & 0.03585 & 0.29270 & 0.25685 \\
\hline $4 \mathrm{f}$ & -0.23140 & 0.03613 & 0.26753 & 0.23140 \\
\hline $4 \mathrm{~g}$ & -0.25814 & 0.09279 & 0.35093 & 0.25814 \\
\hline 4h & -0.25530 & 0.09897 & 0.35427 & 0.2553 \\
\hline $4 \mathbf{i}$ & -0.25411 & 0.07139 & 0.32550 & 0.25411 \\
\hline $4 \mathbf{j}$ & -0.25554 & 0.09192 & 0.34746 & 0.25554 \\
\hline $4 k$ & -0.25663 & 0.09528 & 0.35191 & 0.25663 \\
\hline 41 & -0.25416 & 0.09395 & 0.34811 & 0.25416 \\
\hline $4 m$ & -0.25870 & 0.10473 & 0.36343 & 0.25870 \\
\hline $4 n$ & -0.26027 & 0.08090 & 0.34117 & 0.26027 \\
\hline 40 & -0.25886 & 0.09748 & 0.35634 & 0.25886 \\
\hline $4 p$ & -0.25955 & 0.07268 & 0.33223 & 0.25955 \\
\hline
\end{tabular}




\begin{tabular}{ccccc}
\hline $\mathbf{4 q}$ & -0.26182 & 0.09396 & 0.35578 & 0.26182 \\
$\mathbf{4 r}$ & -0.25921 & 0.09371 & 0.35292 & 0.25921 \\
$\mathbf{T P P}$ & -0.32370 & 0.12855 & 0.45225 & 0.32370 \\
\hline
\end{tabular}

Energies are given in a.u $(1$ a.u $=27.2114 \mathrm{eV}) ; \mathrm{IP}_{\mathrm{KT}}=$ from Koopmans Theorem applied at $\mathrm{HF} / 6-31 \mathrm{G}^{* *} / / \mathrm{B} 3 \mathrm{LYP} / 6-31 \mathrm{G}^{*}$.

\section{Comment:}

DFT has been widely used because the accuracy of the based energy calculation is higher than that based on HF calculation, known to be suitable for examining the electronic structure of LUMO/SOMO due to its clear physical meaning for one-electron molecular orbital (Tsukamoto, K.; Watanabe, T.; Nagashima, U.; Akiyama, Y. J. Mol. Str. (THEOCHEM) 2005, 732, 87). This may be thought to be one of the origins for the relatively significant difference between the predicted IP values at HF and B3LYP levels, to which should be added the fact that different basis sets (6-31G* for B3LYP and 6$31 \mathrm{G}^{* *}$ for HF) were used. Another factor is that all the HF based results stand from Koopman Theorem, which is already know to overestimate the IP, while those based on DFT-B3LYP are known to yield underestimated value.

On the other hand, orbital energy differences are known to be more meaningful in DFT calculations that do not incorporate the Hartree-Fock exchange compared to those calculated with hybrid functionals or the Hartree-Fock method. In HF calculations the virtual orbitals 'see' an $\mathrm{N}$ electron system instead of a N-1 electron system as it should be (Szabo, A.; Ostlund, N. S. Modern Theoretical Chemistry; MacMillan Pub. Inc.: New York, 1982). Therefore, all of these orbitals are too high in energy and are too diffuse. To the extent that HF exchange is incorporated into hybrid DFT this shortcoming is "inherited" from HF in these functionals. There are no observables that can be meaningfully related to the orbital energy differences in either case. By contrast, for "pure" functionals the virtual orbitals are appropriate and the orbital energy differences are well-defined zero-order approximations for excitation energies (Petersilka, M.; Gossmann, U. J.; Gross, E. K. U. Phys. Rev. Lett. 1996, 76, 1212; Petersilka, M.; Gross, E. K. U. Int. J. Quantum Chem. 1996, 30, 181; Görling, A. Phys. Rev. Lett. 1996, 54, 3912). Thus, the discrepancies on the B3LYP and HF predicted LUMO-HOMO ( $\Delta \mathrm{E})$ gap as observed in this work may be seen in this angle. 